\title{
GEOMETRIC CONSEQUENCES OF EXTREMAL BEHAVIOR IN A THEOREM OF MACAULAY
}

\author{
ANNA BIGATTI, ANTHONY V. GERAMITA, AND JUAN C. MIGLIORE
}

\begin{abstract}
F. S. Macaulay gave necessary and sufficient conditions on the growth of a nonnegative integer-valued function which determine when such a function can be the Hilbert function of a standard graded $k$-algebra. We investigate some algebraic and geometric consequences which arise from the extremal cases of Macaulay's theorem. Our work also builds on the fundamental work of G. Gotzmann.

Our principal applications are to the study of Hilbert functions of zeroschemes with uniformity conditions. As a consequence, we have new strong limitations on the possible Hilbert functions of the points which arise as a general hyperplane section of an irreducible curve.
\end{abstract}

\section{INTRODUCTION}

One of the very fruitful ways of studying curves in $\mathbb{P}^{r+1}$ is through a detailed investigation of the points of a general hyperplane section of the curve. This approach has been baptized the Castelnuovo Method in recognition of the early successes of this method achieved by G. Castelnuovo in the early years of this century. A modern introduction to these ideas, along with several notable advances in the method, are reported in the beautiful Montreal lecture notes of D. Eisenbud and J. Harris [EH].

One principal area of investigation has centered on the postulation of the points of a general hyperplane section of a curve in $\mathbb{P}^{r+1}$. Roughly speaking, one shows that the postulation of such point sets has limitations beyond those one might expect. Most frequently, the limitations are the result of the uniformity that all subsets of the hyperplane section (with the same cardinality) must share. One shows that this uniformity is inconsistent with certain postulations since those postulations automatically imply that subsets of the section lie on special varieties while other subsets of the same cardinality (obviously) do not.

In this paper we give many results along these lines, showing how certain types of postulations force very strong geometric conclusions about the set of points. (Actually, many of our results have been stated for higher-dimensional schemes, not even always reduced, although our inspiration was the case of points.) We have tried to greatly generalize some of the known results, and we believe that our techniques should have many further consequences.

The common ingredient of all these results is that they arise because the first

Received by the editors February 1, 1994.

1991 Mathematics Subject Classification. Primary 13D40, 14A05; Secondary 14M99. 
difference of the Hilbert function of that set of points achieves the bound given in a theorem of F. S. Macaulay [M]. This type of extremal behaviour had begun to be studied in related works of G. Gotzmann [Go] and M. Green [Gr], and in fact the latter paper greatly influenced our work.

This paper was motivated by an attempt to understand some results of $\mathrm{E}$. Davis [D] in the context of maximal growth of the Hilbert function. Briefly, Davis' results imply (among other things) that if $Z$ is a zero-dimensional subscheme of $\mathbb{P}^{2}$ whose Hilbert function is of a certain form then $Z$ contains a large subscheme lying on a plane curve of specified degree.

A plane curve is, of course, both a curve and a hypersurface and we have been able to extend the results just noted in both directions.

The extension to hypersurfaces is the easier of the two to establish. We interpret Davis' result about points in $\mathbb{P}^{2}$ as a consequence of one kind of extremal behavior (in the sense of Macaulay) in the growth of the difference function of the Hilbert function of the points and, having isolated that interpretation, we consider that extremal behavior more generally. We prove that a consequence of this behavior (in the special case of the ideal of points in $\mathbb{P}^{n}$ ) is that a large number of the points must lie on a hypersurface of (relatively) low degree. Davis' results for points in $\mathbb{P}^{2}$ are immediate corollaries.

The extension to curves is more subtle. We show (roughly speaking) that if the ideal of a zeroscheme in $\mathbb{P}^{n}$ grows as if it were the ideal of a curve in $\mathbb{P}^{n}$ of degree $s$ then there is a large subscheme actually lying on some curve of degree $s$. Furthermore, under certain hypotheses on the zeroscheme, we show that this curve is reduced and irreducible (see below). We must use the GrusonLazarsfeld-Peskine theorem about the regularity of the ideal sheaf of a reduced and irreducible curve in $\mathbb{P}^{n}$ [GLP] to obtain our results.

We now explain how the paper is organized. In $\S 0$ we review the ingredients from Macaulay's work and the work of Gotzmann and of Green. We then describe (by example) some of the known results which force a large subset of the given points to lie on a variety of some special form, and we give the corresponding conclusions from our results in those cases. The examples are there to show how our results either subsume or extend the known results.

Section 1 lays the groundwork for the results in the later sections. Our situation is different from that of Gotzmann: we assume that the Hilbert function of the quotient ideal $\left(I_{Z}+(L)\right) /(L)$ has maximal growth, and we draw consequences for the original ideal $I_{Z}$. Green has some results along these lines, but we take a different point of view, and we get different sorts of results.

In $\S 2$ we consider the case where a homogeneous component of the ideal (not necessarily of a finite set of points) has a GCD. Here is where we give the “hypersurface" extension of Davis' results about points in $\mathbb{P}^{2}$.

In $\S 3$ we begin the investigation of conditions on the Hilbert function of a set of points in $\mathbb{P}^{n}$ which force a subset to lie on some variety $V$ of dimension $d$, where now $d$ is such that $1 \leq d<n-1$, i.e., we attempt to move beyond the simpler case of $d=n-1$. Although evidence, in the literature, is sparse that there might be such results (although see [Ma, Lemma 2.2 and Theorem 2.3] for some noteworthy examples) we are able to get rather strong conclusions for the case $d=1$ (i.e., for $V$ a curve in $\mathbb{P}^{n}$ ). Our main results in this section (Theorem 3.6 and Corollary 3.7 ) show that not only is $V$ a curve, but if the 
set of points is reduced then so is $V$. Furthermore, in this case a great deal of information is obtained about the relations between the subset of points lying on $V$ and the subset off $V$.

For $1<d<n-1$ our results are less complete. We do, however, get some interesting results for the case of $V$ a linear subvariety or $V$ a hypersurface in a linear subvariety. In this way we recapture one result of Green [Gr, Theorem 3] (our Lemma 3.1), with a completely different proof, and slightly extend another [Gr, Theorem 4] (our Corollary 3.2).

In $\S 4$ we give some applications of our results. The most striking ones eliminate certain Hilbert functions as the Hilbert function of the general hyperplane section of a reduced and irreducible curve in $\mathbb{P}^{n}(n>3)$. These are very different from the restrictions first established by Eisenbud and Harris [EH]. We do this by showing how maximal growth together with certain uniformity assumptions on the set of points have strong consequences on $V$ and on the number of points lying on $V$, when $V$ is a hypersurface and when $V$ is a curve. In the latter case, for instance, Theorem 4.2 shows that if the points are in linear general position and $s<2 n$ then $V$ is irreducible and "almost all" of the points lie on $V$. More strikingly, in Theorem 4.7 we show that if the points have the Uniform Position Property (with no assumption on $s$ ) then again $V$ is irreducible and now all of the points lie on $V$.

We are grateful to Y. Pitteloud for reminding us about the paper [Gr], which was instrumental in leading us to the approach we have taken in this paper. The first and third authors are very grateful to the Department of Mathematics and Statistics of Queen's University for their hospitality. The first author would like to thank the Italian C.N.R., and the second and third authors would like to thank the N.S.E.R.C. of Canada, for financial support during the preparation of this work.

\section{BACKGROUND RESULTS}

Let $R=k\left[X_{0}, \ldots, X_{n}\right]$ be the polynomial ring in $n$ variables over the field $k$, with its usual gradation. Let $I \subseteq R$ be a homogeneous ideal in $R$ and set $A=R / I$. Then $A=\bigoplus A_{t}(t \geq 0)$ has an obvious gradation where $A_{t}=R_{t} / I_{t}$. The Hilbert function of $A$ is the integer valued function on the natural numbers denoted by

$$
H(A, t):=\operatorname{dim}_{k} A_{t}=\operatorname{dim}_{k} R_{t}-\operatorname{dim}_{k} I_{t}=\left(\begin{array}{c}
t+n \\
n
\end{array}\right)-\operatorname{dim}_{k} I_{t} .
$$

The first difference of the function $H$ above is denoted $\Delta H$ and is defined by

$$
\Delta H(A, t):=H(A, t)-H(A, t-1) .
$$

The ideal $m=\left(X_{0}, \ldots, X_{n}\right) \subseteq R$ is usually called the irrelevant maximal ideal of $R$ and its image in $A, \bar{m}$, is called the irrelevant maximal ideal of $A$.

Recall that a saturated homogeneous ideal $I$ of the graded ring $A$ is a homogeneous ideal in $A$ for which the irrelevant maximal ideal of $A$ is not an associated prime ideal. For any homogeneous ideal $I$ of $A$, the saturation of $I$, denoted $I^{\text {sat }}$, is defined by

$$
I^{\text {sat }}:=\left\{f \in A: f \bar{m}^{l} \subseteq I \text { for some integer } l\right\} .
$$

It is well known that for any homogeneous ideal $I$ one has $I_{t}=\left(I^{\text {sat }}\right)_{t}$ for all $t \gg 0$. 
If $I$ is the saturated ideal of some subscheme $\mathbb{X} \subseteq \mathbb{P}^{n}$ and $A=R / I$ is the homogeneous coordinate ring of $\mathbb{X}$ then we sometimes write $H(\mathbb{X}, t)$ (or even $\left.H_{\mathrm{X}}(t)\right)$ for $H(A, t)$ and refer to this Hilbert function as the Hilbert function of $\mathbb{X}$.

It is easy to see that not any function can arise as the Hilbert function of a ring such as $A$ above but the precise constraints on such a function seem obscure. Fortunately, such functions are well classified, thanks to a remarkable theorem of F. S. Macaulay [M]. Since we shall need some of the features of Macaulay's classification (and also G. Gotzmann's significant elaborations of Macaulay's work) we shall take some time to explain a few of their main ideas here.

For the reader interested in more details on Macaulay's Theorem, we recommend either the lucid paper of R. Stanley [S] or the strikingly elementary proof of the theorem given by M. Green in [Gr]. Gotzmann's results are proved in [Go]. The reader interested in an English language proof can consult the paper of Green referred to above.

We begin with a discussion of Macaulay's Theorem which describes the nature of the growth of the Hilbert function. Recall that Hilbert had already proved that if $A$ is a standard graded $k$-algebra (i.e., a graded Noetherian $k$ algebra generated-as a $k$-algebra-by its homogeneous elements of degree 1) and if $H(A, t)=a_{t}$ then there is a polynomial $f(x) \in \mathbb{Q}[x]$ such that for all $t \gg 0, f(t)=a_{t}$. This polynomial (called the Hilbert polynomial of $A$ ) determines the eventual growth of the Hilbert function. One of the more beautiful chapters of commutative algebra explains how the coefficients of this polynomial carry information about $A$. For the moment, we shall only note that

$$
\operatorname{deg} f(x)=(\text { Krull dimension } A)-1
$$

(where the zero polynomial will, by convention, have degree -1 ).

Macaulay explains the growth of the Hilbert function in terms of the so-called $i$-binomial expansion, so we shall first recall that notion.

Definition-Propositon 0.1. The $i$-binomial expansion of the integer $h(i, h>0)$ is the unique expression

$$
h=\left(\begin{array}{c}
m_{i} \\
i
\end{array}\right)+\left(\begin{array}{c}
m_{i-1} \\
i-1
\end{array}\right)+\cdots+\left(\begin{array}{c}
m_{j} \\
j
\end{array}\right),
$$

where $m_{i}>m_{i-1}>\cdots>m_{j} \geq j \geq 1$.

Example 0.2. The 4-binomial expansion of 85 is

$$
85=\left(\begin{array}{l}
8 \\
4
\end{array}\right)+\left(\begin{array}{l}
5 \\
3
\end{array}\right)+\left(\begin{array}{l}
3 \\
2
\end{array}\right)+\left(\begin{array}{l}
2 \\
1
\end{array}\right)
$$

We now define a collection of functions ${ }^{i i\rangle}: \mathbb{Z} \rightarrow \mathbb{Z}$ as follows: if $h \in \mathbb{Z}$ has $i$-binomial expansion as above, then

$$
h^{\langle i\rangle}=\left(\begin{array}{c}
m_{i}+1 \\
i+1
\end{array}\right)+\left(\begin{array}{c}
m_{i-1}+1 \\
i
\end{array}\right)+\cdots+\left(\begin{array}{c}
m_{j}+1 \\
j+1
\end{array}\right) .
$$

So, for example, $85^{\langle 4\rangle}=\left(\begin{array}{l}9 \\ 5\end{array}\right)+\left(\begin{array}{l}6 \\ 4\end{array}\right)+\left(\begin{array}{l}4 \\ 3\end{array}\right)+\left(\begin{array}{l}3 \\ 2\end{array}\right)=148$. 
Definition 0.3. A sequence of nonnegative integers $\left\{c_{i}: i \geq 0\right\}$ is called an $O$-sequence (" $O$ " is for the letter "oh"), if

$$
c_{0}=1 \text { and } c_{i+1} \leq c_{i}^{\langle i\rangle}, \text { for all } i .
$$

Theorem 0.4 (Macaulay [M]). The following are equivalent:

(a) $\left\{c_{i}: i \geq 0\right\}$ is an O-sequence;

(b) $\left\{c_{i}: i \geq 0\right\}$ is the Hilbert function of a standard graded $k$-algebra.

Gotzmann's elaboration of Macaulay's Theorem is also very interesting and deals with the extremal situation described by Macaulay's growth condition.

To fix the notation, let $R$ be as above and suppose $W$ is a subspace of $R_{d}$ of/codimension $b_{d}$. Write the $d$-binomial expansion of $b_{d}$ as

$$
b_{d}=\left(\begin{array}{c}
m_{d} \\
d
\end{array}\right)+\left(\begin{array}{c}
m_{d-1} \\
d-1
\end{array}\right)+\cdots+\left(\begin{array}{c}
m_{j} \\
j
\end{array}\right)
$$

where $m_{d}>m_{d-1}>\cdots>m_{j} \geq j \geq 1$.

Let $J$ be the ideal of $R$ generated by $W$ and write $B=R / J$. Then $H(B, d)=b_{d}$ and so, by Macaulay's Theorem, $H(B, d+1)=b_{d+1} \leq b_{d}^{\langle d\rangle}$. Thus, $\operatorname{dim}_{k} J_{d+1}=\operatorname{dim}_{k}\left(R_{1} J_{d}\right) \geq\left(\begin{array}{c}d+1+n \\ n\end{array}\right)-b_{d}^{\langle d\rangle}$. Hence $W$ grows least when $\operatorname{dim}_{k} J_{d+1}=\left(\begin{array}{c}d+1+n \\ n\end{array}\right)-b_{d}^{\langle d\rangle}$, i.e., when $b_{d+1}=b_{d}^{\langle d\rangle}$. Gotzmann's Persistence Theorem has a great deal to say in this latter situation.

Theorem 0.5 (Gotzmann [Go]). Let $W, J$ and $b_{d}$ be as above and suppose that $b_{d+1}=b_{d}^{\langle d\rangle}$. Then, for any $l \geq 1$ we have:

$$
b_{d+l}=\left(\begin{array}{c}
m_{d}+l \\
d+l
\end{array}\right)+\left(\begin{array}{c}
m_{d-1}+l \\
d-1+l
\end{array}\right)+\cdots+\left(\begin{array}{c}
m_{j}+l \\
j+l
\end{array}\right) .
$$

In other words, the Hilbert Polynomial of $B=R / J$ is

$$
\begin{aligned}
p(x) & =\left(\begin{array}{c}
m_{d}+x-d \\
x
\end{array}\right)+\left(\begin{array}{c}
m_{d-1}+x-d \\
x-1
\end{array}\right)+\cdots+\left(\begin{array}{c}
m_{j}+x-d \\
x-d+j
\end{array}\right) \\
& =\left(\begin{array}{c}
x+m_{d}-d \\
m_{d}-d
\end{array}\right)+\left(\begin{array}{c}
x+m_{d-1}-d \\
m_{d-1}-(d-1)
\end{array}\right)+\cdots+\left(\begin{array}{c}
x+m_{j}-d \\
m_{j}-j
\end{array}\right),
\end{aligned}
$$

where $m_{d}-d \geq m_{d-1}-d+1 \geq \cdots \geq m_{j}-j \geq 0$.

Remark 0.6. We shall have occasion, later, to make some observations about the Hilbert polynomial guaranteed by Gotzmann's Persistence Theorem and it seems worthwhile to note a few things about that polynomial here.

Let $W, J$, and $b_{d}$ be as above. Since $\left(\begin{array}{l}x \\ a\end{array}\right)$ is a polynomial of degree $a$ with top coefficient $1 / a$ ! we see that the Hilbert polynomial of $R / J$ has degree $m_{d}-d$. So, if $\mathbb{X}$ is the variety defined by $J^{\text {sat }}$, then the top-dimensional component of $\mathbb{X}$ has dimension $m_{d}-d$.

Now notice that $m_{d}-d=m_{i}-i$ for $(j \leq i<d)$ if and only if $m_{i}=$ $m_{d}-(d-i)$. In view of the restriction on the $m_{t}$ in the definition of the $d$-binomial expansion, we must then have

$$
m_{d-1}=m_{d}-1 ; m_{d-2}=m_{d-1}-1 ; \cdots ; m_{i}=m_{i+1}-1 .
$$

So, the top coefficient of the Hilbert polynomial is $\left(s /\left(m_{d}-d\right) !\right)$ if and only if $m_{d-(s-1)}=m_{d}-(s-1)$ and $m_{d-s}<m_{d}-s$. 
If we order all the possible Hilbert polynomials by saying that $f(x)<g(x)$ if there is an integer $N$ so that for all $x_{0} \geq N$ we have $f\left(x_{0}\right)<g\left(x_{0}\right)$, then we see that the least polynomial that can be the Hilbert polynomial of a subscheme of $\mathbb{P}^{n}$ having dimension $d$ and degree $s$ is

$$
\left(\begin{array}{l}
x \\
d
\end{array}\right)+\left(\begin{array}{c}
x+1 \\
d
\end{array}\right)+\cdots+\left(\begin{array}{c}
x+s-1 \\
d
\end{array}\right) \text {. }
$$

This means that for all $t \gg 0$ we must have

$$
b_{t}=\left(\begin{array}{c}
t+d \\
t
\end{array}\right)+\left(\begin{array}{c}
t-1+d \\
t-1
\end{array}\right)+\cdots+\left(\begin{array}{c}
t-(s-1)+d \\
t-(s-1)
\end{array}\right) .
$$

(See Lemma 2.5 below.)

We have, for some time, been aware of a collection of results about the Hilbert function of a set of points which all have, as consequence, that the set of points has a distinguished subset lying on a distinguished subvariety. Rather than trying to state this entire collection of results in their full generality, we shall illustrate some of the more notable theorems with some examples.

Example 0.7 (Castelnuovo). Let $\mathbb{X}$ be a set of 30 points in $\mathbb{P}^{5}$ with Hilbert function

$$
\begin{array}{llllll}
1 & 6 & 21 & 25 & 30 & 30 \ldots
\end{array}
$$

and thus having first difference

$$
\begin{array}{llllll}
1 & 5 & 15 & 4 & 5 & 0 .
\end{array}
$$

An elementary result, attributed by Maroscia (see [Ma, Lemma 2.1]) to G. Castelnuovo, says that 6 points $\mathbb{X}$ lie on a $\mathbb{P}^{4} \subset \mathbb{P}^{5}$. Our Theorem 3.3 says, instead, that 15 points of $\mathbb{X}$ lie on a $\mathbb{P}^{2} \subset \mathbb{P}^{5}$. If we use $c_{i}$ to denote the values of the difference function, note that the passage from $c_{3}$ to $c_{4}$ is the maximum permitted by Macaulay's Theorem.

Example 0.8 (E. D. Davis). Let $\mathbb{X}$ be a set of 21 points in $\mathbb{P}^{2}$ with Hilbert function

$$
\begin{array}{lllllllllll}
1 & 3 & 6 & 10 & 14 & 16 & 18 & 20 & 21 & 21 \ldots,
\end{array}
$$

i.e., with first difference

$$
\begin{array}{llllllllll}
1 & 2 & 3 & 4 & 4 & 2 & 2 & 2 & 1 & 0 .
\end{array}
$$

Then $\mathbb{X}$ contains a subset of 16 points lying on a conic in $\mathbb{P}^{2}$. (See [D, Theorem 4.1].) Our Corollary 2.10 or Theorem 3.6 can be applied to this example to give the same result. Since Davis' theorem is the strongest possible result for $\mathbb{P}^{2}$ our results can only capture his for $\mathbb{P}^{2}$. If we use $c_{i}$ to denote the values of the difference function, note that the passage from $c_{5}$ to $c_{6}$ is the maximum permitted by Macaulay's Theorem.

Example 0.9 (A. V. Geramita, P. Maroscia, L. G. Roberts). Let $\mathbb{X}$ be a set of points in $\mathbb{P}^{3}$ with Hilbert function

$$
\begin{array}{llllllll}
1 & 4 & 10 & 15 & 16 & 17 & 18 & 18 \ldots,
\end{array}
$$

i.e., with first difference 
Then $\mathbb{X}$ has a subset of 7 points on a $\mathbb{P}^{1} \subset \mathbb{P}^{3}$. (See [GMR, Proposition 5.2].) Our Theorem 3.3 or 3.6 has this result as a special case. Again, using $c_{i}$ to denote the values of the difference function, we see that the passage from $c_{4}$ to $c_{5}$ is the maximum permitted by Macaulay's Theorem.

Example 0.10 (P. Maroscia). Let $\mathbb{X}$ be a set of 26 points in $\mathbb{P}^{4}$ with Hilbert function

$$
\begin{array}{llllllll}
1 & 5 & 15 & 18 & 21 & 24 & 26 & 26 \ldots,
\end{array}
$$

i.e., whose Hilbert function has first difference

$$
\begin{array}{llllllll}
1 & 4 & 10 & 3 & 3 & 3 & 2 & 0 .
\end{array} .
$$

Then the Castelnuovo result referred to in Example 0.6 implies that at least 5 points of $\mathbb{X}$ must lie on a $\mathbb{P}^{3} \subseteq \mathbb{P}^{4}$. Maroscia [Ma, Theorem 2.3] asserts that, in the presence of a small amount of uniformity-in this case, no 4 of the points of $\mathbb{X}$ lie on a $\mathbb{P}^{2}$-then at least 17 of the 26 points of $\mathbb{X}$ lie on a rational normal curve in a $\mathbb{P}^{3} \subseteq \mathbb{P}^{4}$.

Unfortunately, Maroscia's arguments require the long string of 3 's in the difference function above.

On the other hand, our Theorem 4.2 gives that 18 of the 26 points of $\mathbb{X}$ must lie on a rational normal curve in a $\mathbb{P}^{3} \subseteq \mathbb{P}^{4}$. Moreover, we get the same result for a set of points in $\mathbb{P}^{4}$ whose Hilbert function has first difference

$$
\begin{array}{llllllll}
1 & 4 & 10 & 4 & 3 & 3 & 2 & 0
\end{array}
$$

(an example to which Maroscia's Theorem does not apply).

Example 0.11. Let $X$ be a set of 104 points in $\mathbb{P}^{3}$. Suppose that the Hilbert function of this set is

$$
\begin{array}{llllllllllll}
1 & 4 & 10 & 20 & 34 & 48 & 64 & 79 & 96 & 100 & 104 & 104 \ldots
\end{array}
$$

and that no 4 of the points lie on a plane.

The results we shall prove will show that exactly 89 of these points lie on a quadric surface and of those, either 40 or 41 (both cases are possible) lie on an irreducible curve of degree 4 (see Theorem 4.2).

The remaining $104-89=15$ points have exactly 13 of them lying in a rational normal curve in $\mathbb{P}^{3}$.

One of our main goals in this paper is to explain how all the results illustrated above (and many more) can be viewed as arising from extremal behavior in Macaulay's Theorem.

\section{Fundamental CONSEQUeNCES OF MAXIMAL GROWTH OF THE HILBERT FUNCTION OF SATURATED IDEALS}

As we noted in the previous section, there are some striking consequences of maximal growth of the Hilbert function at some place. In this section we will collect some fundamental consequences of maximal growth that we have observed for the Hilbert function of saturated ideals. Although of interest in their own right, they will also play an important role in the results to follow.

In order to simplify later discussions, we introduce the following terminology:

Let $\mathfrak{A}$ be a homogeneous ideal in the polynomial ring $\mathbf{P}$ and let $\mathbf{B}=\mathbf{P} / \mathfrak{A}$. If $b_{i}=H(\mathbf{B}, i)$ is the Hilbert function of the ring $\mathbf{B}$ in degree $i$ then we say 
B has maximal growth in degree $d$ if $b_{d+1}=b_{d}^{\langle d\rangle}$ (see the discussion before 0.3 ).

If $T$ is a subset of the ring $\mathbf{P}$ we use the notation $\langle T\rangle$ to indicate the ideal in $\mathbf{P}$ generated by the set $T$.

We will follow the standard notation and if $\mathscr{F}$ denotes a coherent sheaf on the scheme $\mathbb{X}$ we use $h^{i}(\mathscr{F})$ to denote the vector space dimension of the cohomology group $H^{i}(\mathscr{F})$.

Lemma 1.1. Let $I$ be a saturated ideal in the polynomial ring $R=k\left[X_{0}, \ldots, X_{n}\right]$ and let $L$ be a linear form in $R$ which is not a zero divisor on the ring $A=R / I$. Let $J$ be the ideal $I / L I \simeq(I, L) /(L)$ in the polynomial ring $S=R /(L)$ and set $B=S / J$.

If $A$ has maximal growth in degree $d$ then $B$ also has maximal growth in degree $d$.

Proof. Let $H(A, d)=a_{d}=\left(\begin{array}{c}m_{d} \\ d\end{array}\right)+\left(\begin{array}{c}m_{d-1} \\ d-1\end{array}\right)+\cdots+\left(\begin{array}{c}m_{i} \\ j\end{array}\right)$ be the $d$-binomial expansion of $a_{d}$. Then, since $A$ has maximal growth in degree $d$ we have

$$
H(A, d+1)=a_{d+1}=a_{d}^{\langle d\rangle}=\left(\begin{array}{c}
m_{d}+1 \\
d+1
\end{array}\right)+\left(\begin{array}{c}
m_{d-1}+1 \\
d
\end{array}\right)+\cdots+\left(\begin{array}{c}
m_{j}+1 \\
j+1
\end{array}\right) .
$$

Since $L$ is not a zero divisor on $A$, the difference function of the Hilbert function of $A$ is the Hilbert function of $B$. Thus,

$$
\begin{aligned}
H(B, d+1) & =b_{d+1}=a_{d+1}-a_{d} \\
& =\left[\left(\begin{array}{c}
m_{d}+1 \\
d+1
\end{array}\right)-\left(\begin{array}{c}
m_{d} \\
d
\end{array}\right)\right]+\cdots+\left[\left(\begin{array}{c}
m_{j}+1 \\
j+1
\end{array}\right)-\left(\begin{array}{c}
m_{j} \\
j
\end{array}\right)\right] \\
& =\left(\begin{array}{c}
m_{d} \\
d+1
\end{array}\right)+\left(\begin{array}{c}
m_{d-1} \\
d
\end{array}\right)+\cdots+\left(\begin{array}{c}
m_{j} \\
j+1
\end{array}\right) .
\end{aligned}
$$

Now, suppose that $B$ does not have maximal growth in degree $d$, i.e., $b_{d+1}<\left(b_{d}\right)^{\langle d\rangle}$. Then, since

$$
b_{d+1}=\left(\left(\begin{array}{c}
m_{d}-1 \\
d
\end{array}\right)+\left(\begin{array}{c}
m_{d-1}-1 \\
d-1
\end{array}\right)+\cdots+\left(\begin{array}{c}
m_{j}-1 \\
j
\end{array}\right)\right)^{\langle d\rangle}
$$

we must have

$$
b_{d}>\left(\begin{array}{c}
m_{d}-1 \\
d
\end{array}\right)+\left(\begin{array}{c}
m_{d-1}-1 \\
d-1
\end{array}\right)+\cdots+\left(\begin{array}{c}
m_{j}-1 \\
j
\end{array}\right) .
$$

Note that

$$
\begin{aligned}
a_{d-1}= & a_{d}-b_{d}<\left(\left(\begin{array}{c}
m_{d} \\
d
\end{array}\right)+\left(\begin{array}{c}
m_{d-1} \\
d-1
\end{array}\right)+\cdots+\left(\begin{array}{c}
m_{j} \\
j
\end{array}\right)\right) \\
& -\left(\left(\begin{array}{c}
m_{d}-1 \\
d
\end{array}\right)+\left(\begin{array}{c}
m_{d-1}-1 \\
d-1
\end{array}\right)+\cdots+\left(\begin{array}{c}
m_{j}-1 \\
j
\end{array}\right)\right) \\
= & \left(\begin{array}{c}
m_{d}-1 \\
d-1
\end{array}\right)+\left(\begin{array}{c}
m_{d-1}-1 \\
d-2
\end{array}\right)+\cdots+\left(\begin{array}{c}
m_{j}-1 \\
j-1
\end{array}\right) .
\end{aligned}
$$

There are now two simple cases to consider: 
If $j>1$ : Then $j-1>0$ and the bound above for $a_{d-1}$ is exactly the $(d-1)$-binomial expansion of $a_{d-1}$. By Macaulay's Theorem 0.4 we then have

$$
\begin{aligned}
a_{d} & \leq a_{d-1}^{\langle d-1\rangle}<\left(\left(\begin{array}{c}
m_{d}-1 \\
d-1
\end{array}\right)+\left(\begin{array}{c}
m_{d-1}-1 \\
d-2
\end{array}\right)+\cdots+\left(\begin{array}{c}
m_{j}-1 \\
j-1
\end{array}\right)\right)^{\langle d-1\rangle} \\
& =\left(\begin{array}{c}
m_{d} \\
d
\end{array}\right)+\left(\begin{array}{c}
m_{d-1} \\
d-1
\end{array}\right)+\cdots+\left(\begin{array}{c}
m_{j} \\
j
\end{array}\right)=a_{d}
\end{aligned}
$$

which is a contradiction.

If $j=1$ : Then $\left(\begin{array}{c}m_{j}-1 \\ j-1\end{array}\right)=1$, and this implies that

$$
a_{d-1} \leq\left(\begin{array}{c}
m_{d}-1 \\
d-1
\end{array}\right)+\left(\begin{array}{c}
m_{d-1}-1 \\
d-2
\end{array}\right)+\cdots+\left(\begin{array}{c}
m_{j+1}-1 \\
j
\end{array}\right) .
$$

By Macaulay's Theorem 0.4 we thus have

$$
\begin{aligned}
a_{d} & \leq\left(\left(\begin{array}{c}
m_{d}-1 \\
d-1
\end{array}\right)+\left(\begin{array}{c}
m_{d-1}-1 \\
d-2
\end{array}\right)+\cdots+\left(\begin{array}{c}
m_{j+1}-1 \\
j
\end{array}\right)\right)^{\langle d-1\rangle} \\
& =\left(\begin{array}{c}
m_{d} \\
d
\end{array}\right)+\left(\begin{array}{c}
m_{d-1} \\
d-1
\end{array}\right)+\cdots+\left(\begin{array}{c}
m_{j+1} \\
j+1
\end{array}\right)<a_{d}
\end{aligned}
$$

which is again a contradiction.

Remark 1.2. Notice that, in the lemma above, the Hilbert function of the ring $B$, in degree $d+1$, is the minimum permitted by a theorem of Green [Gr, Theorem 1]. L. Robbiano has pointed out to us that a shorter argument for Lemma 1.1 can be given using the calculus for binomial expansions which he worked out in [Ro]. We have retained our proof since it is self-contained.

Examples 1.3. There are two examples related to Lemma 1.1 that we found of interest. They illustrate some of the limitations surrounding obvious extensions of the statement of that lemma.

(a) Consider the set of 8 points, $\mathbb{X}$, in $\mathbb{A}^{2} \subset \mathbb{P}^{2}$, situated as follows:

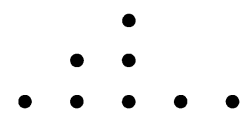

The ideal $I=I_{\mathrm{X}} \subseteq k\left[X_{0}, X_{1}, X_{2}\right]=R$ of these points is a saturated ideal and $A=R / I$ has Hilbert function

$$
\begin{array}{lllllll}
1 & 3 & 6 & 7 & 8 & 8 \ldots
\end{array}
$$

If $L$ is the equation of a line in $\mathbb{P}^{2}$ missing the points of $\mathbb{X}$, then $L$ is not a zero divisor in $A$. So, $A / \bar{L} A=B$ has Hilbert function which is the difference function of the Hilbert function of $A$, i.e.,

$$
\begin{array}{lllllll}
1 & 2 & 3 & 1 & 1 & 0 \ldots
\end{array}
$$

Clearly $B$ has maximal growth in degree 3 . Notice, however, that $A$ does not have maximal growth in degree 3 since the 3-binomial expansion of 7 is $\left(\begin{array}{l}4 \\ 3\end{array}\right)+\left(\begin{array}{l}3 \\ 2\end{array}\right)$ and so $7^{\langle 3\rangle}=\left(\begin{array}{l}5 \\ 4\end{array}\right)+\left(\begin{array}{l}4 \\ 3\end{array}\right)=9>8$.

(b) There are many saturated ideals $I \subseteq k\left[X_{0}, X_{1}, X_{2}, X_{3}\right]=R$ for which $A=R / I$ has Hilbert function beginning 146 . Since the 2-binomial expansion of 6 is $6=\left(\begin{array}{l}4 \\ 2\end{array}\right)$ and since $6^{\langle 2\rangle}=\left(\begin{array}{l}5 \\ 3\end{array}\right)=10$ there are also ideals $I^{\prime}$ in $R$ whose 
Hilbert function begins 14610 , i.e., ideals whose Hilbert function has maximal growth in degree 2 . However, no such ideal $I^{\prime}$ can be saturated.

To see why this is so, suppose we had such a saturated ideal $I^{\prime}$. We would then be able to find a linear form $L$ in $R$ which was not a zero divisor on the ring $A=R / I^{\prime}$. Then $B=A / \bar{L} A$ would be a ring whose Hilbert function began $\begin{array}{lllll} & 3 & 2 & 4 & \ldots\end{array}$. Since such a sequence is not an $O$-sequence, this is impossible.

Lemma 1.4. Let $I \subset R=k\left[X_{0}, \ldots, X_{n}\right]$ be a saturated ideal and let $L \in R_{1}$ be a general linear form. Let $J=I /(L I) \simeq(I, L) /(L) \subset R /(L)=S$. Suppose $S / J$ has maximal growth in degree $d$.

Let $\bar{I}=\left\langle I_{\leq d}\right\rangle$ and let $\tilde{I}=\bar{I}^{\text {sat }}$ be the saturation of the ideal $\bar{I}$. Then $\bar{I}=\tilde{I}$, i.e., $\bar{I}$ is a saturated ideal.

Proof. It suffices to show that the two homogeneous ideals agree in every degree.

Define $\bar{J}=\left\langle J_{\leq d}\right\rangle$. Then clearly,

$$
\bar{J}=(\bar{I}+L I) / L I \simeq \bar{I} /(\bar{I} \cap L I) .
$$

Since $L$ is not a zero divisor modulo $I$, we have $L I=(L) \cap I$. Hence,

$$
\bar{I} \cap L I=\bar{I} \cap(L) \cap I=(L) \cap \bar{I} \quad(\text { since } \bar{I} \subseteq I) .
$$

This gives

$$
\bar{J}=\bar{I} /((L) \cap \bar{I}) \simeq(\bar{I}+(L)) /(L) .
$$

Now $L \bar{I} \subseteq(L) \cap \bar{I}$ and so we have a surjection

$$
\bar{I} / L \bar{I} \rightarrow \bar{I} /(L) \cap \bar{I}=\bar{J} .
$$

Thus, since $L$ is not a zero divisor in $R$,

$$
\operatorname{dim} \bar{I}_{i}-\operatorname{dim} \bar{I}_{i-1} \geq \operatorname{dim} \bar{J}_{i}
$$

for every integer $i \geq 1$.

Since $\tilde{I}$ is the saturation of $\bar{I}$ we have, for all $t \gg 0$, that $\bar{I}_{t}=\tilde{I}_{t}$. We shall assume that this is the case for all $t \geq N$.

Hence, for $t \geq N+1$,

$$
L \bar{I}_{t-1}=(L \bar{I})_{t}=((L) \cap \bar{I})_{t},
$$

i.e.,

$$
\operatorname{dim} \bar{I}_{t-1}=\operatorname{dim}((L) \cap \bar{I})_{t}
$$

Since for any $t$ whatsoever we have

$$
\operatorname{dim} \bar{J}_{t}=\operatorname{dim} \bar{I}_{t}=\operatorname{dim}((L) \cap \bar{I})_{t}
$$

we obtain, for $t \geq N+1$,

$$
\operatorname{dim} \bar{J}_{t}=\operatorname{dim} \bar{I}_{t}-\operatorname{dim} \bar{I}_{t-1}=\operatorname{dim} \tilde{I}_{t}-\operatorname{dim} \tilde{I}_{t-1} .
$$

Now $\bar{I} \subseteq \tilde{I}$ (in general) but now choose $F \in \tilde{I}(\operatorname{deg} F \leq d)$. Then $F m^{l} \subseteq \bar{I}$ for some integer $l$ and $m$ the irrelevant maximal ideal of $R$. Since $\bar{I} \subseteq I$ we thus have $F m^{l} \subseteq I$ and so $F \in I^{\text {sat }}$. Since $I$ is already saturated we conclude that $F \in I$. Since $I$ and $\bar{I}$ agree in degree $\leq d$ we obtain $\bar{I}_{t}=\tilde{I}_{t}$ for all $t \leq d$. 
Since $S / J$ has maximal growth in degree $d$, this implies that $J$ has no minimal generator in degree $d+1$. Since $L$ was not a zero divisor modulo $I$, this implies that $I$ has no minimal generator in degree $d+1$, i.e., $I_{d}$ generates $I_{d+1}$. Thus $\bar{I}$ and $\tilde{I}$ also agree in degree $d+1$.

So we have

$$
\begin{aligned}
\bar{I}_{d-1} & =\tilde{I}_{d-1} \\
\bar{I}_{d} & =\tilde{I}_{d} \\
\bar{I}_{d+1} & =\tilde{I}_{d+1} \\
\bar{I}_{d+2} & \subseteq \tilde{I}_{d+2} \\
& \cdots \\
& \cdots \\
\bar{I}_{N} & =\tilde{I}_{N} \\
\bar{I}_{N+1} & =\tilde{I}_{N+1}
\end{aligned}
$$

Now consider the successive differences in the two columns above. We have

$$
\begin{gathered}
\operatorname{dim} \bar{J}_{d}=\operatorname{dim} \bar{I}_{d}-\operatorname{dim} \bar{I}_{d-1}=\operatorname{dim} \tilde{I}_{d}-\operatorname{dim} \tilde{I}_{d-1} \\
\operatorname{dim} \bar{J}_{d+1}=\operatorname{dim} \bar{I}_{d+1}-\operatorname{dim} \bar{I}_{d}=\operatorname{dim} \tilde{I}_{d+1}-\operatorname{dim} \tilde{I}_{d} \\
\operatorname{dim} \bar{J}_{d+2} \leq \operatorname{dim} \bar{I}_{d+2}-\operatorname{dim} \bar{I}_{d+1} \leq \operatorname{dim} \tilde{I}_{d+2}-\operatorname{dim} \tilde{I}_{d+1} \\
\ldots \\
\ldots \\
\operatorname{dim} \bar{J}_{N+1}=\operatorname{dim} \bar{I}_{N+1}-\operatorname{dim} \bar{I}_{N}=\operatorname{dim} \tilde{I}_{N+1}-\operatorname{dim} \tilde{I}_{N}
\end{gathered}
$$

Let $i$ be the first integer for which

$$
\operatorname{dim} \bar{J}_{i}<\operatorname{dim} \tilde{I}_{i}-\operatorname{dim} \tilde{I}_{i-1} .
$$

Then $i \geq d+2$ and, from the definition of $i$ we have

$$
\begin{aligned}
H(R / \bar{I}, i-2) & =H(R / \tilde{I}, i-2) \\
H(R / \bar{I}, i-1) & =H(R / \tilde{I}, i-1) \\
H(R / \bar{I}, i) & >H(R / \tilde{I}, i) \\
& \cdots \\
H(R / \bar{I}, N) & =H(R / \tilde{I}, N) \\
H(R / \bar{I}, N+1) & =H(R / \tilde{I}, N+1)
\end{aligned}
$$


Thus,

$$
\begin{aligned}
\Delta H(R / \bar{I}, i-1)=\Delta H(R / \tilde{I}, i-1) & =H(S / \bar{J}, i-1) \\
\Delta H(R / \tilde{I}, i) & <H(S / \bar{J}, i) \\
\cdots & \cdots \\
\cdots & \cdots(S / \bar{J}, N+1) \\
\Delta H(R / \tilde{I}, N+1) & =H(S / \bar{J}, N+2) \\
\Delta H(R / \tilde{I}, N+2) & =H(S, N)
\end{aligned}
$$

Since $\tilde{I}$ is a saturated ideal and $L$ is not a zero divisor modulo $\tilde{I}$, we have that $\Delta H(R / \tilde{I},-)$ is the Hilbert function of the quotient of the ring $S$ by the ideal $(\bar{I}, L) /(L)$. But, by construction, $S / \bar{J}$ has maximal growth (by the Gotzmann Persistence Theorem) for all $j \geq d$. So, in particular, this is true for all $j \geq i-1$. But $\Delta H(R / \tilde{I},-)$ falls behind this maximal growth in degree $i$ and hence can never catch up! The fact that it does catch up in degree $N+1$ is the required contradiction.

Thus,

$$
\operatorname{dim} \bar{I}_{t}-\operatorname{dim} \bar{I}_{t-1}=\operatorname{dim} \tilde{I}_{t}-\operatorname{dim} \tilde{I}_{t-1}
$$

for every $t$. Since $\bar{I}$ and $\tilde{I}$ agree in low degrees, this implies that they agree in all degrees, as we wanted to show.

There is more than can be said about the ideal $\bar{I}=\tilde{I}$ of Lemma 1.4. To explain this, recall a definition originally due to Mumford [Mu].

A coherent sheaf $\mathscr{F}$ on $\mathbb{P}^{n}$ is said to be $s$-regular if $H^{q}(\mathscr{F}(s-q))=0$ for all $q>0$. The least integer $r$ for which $\mathscr{F}$ is $r$-regular is called the regularity of $\mathscr{F}$.

In his paper [Go], Gotzmann found a very strong connection between regularity and the Hilbert polynomial. We recall Gotzmann's theorem here. (An English language proof, which slightly improves Gotzmann's theorem, can be found in [Gr].)

Theorem 1.5 (Gotzmann Regularity Theorem). Any graded ideal $I \subseteq R=$ $k\left[X_{0}, \ldots, X_{n}\right]$ has Hilbert polynomial which can be uniquely written in the form

$$
p(x)=\left(\begin{array}{c}
x+a_{1} \\
a_{1}
\end{array}\right)+\left(\begin{array}{c}
x+a_{2}-1 \\
a_{2}
\end{array}\right)+\cdots+\left(\begin{array}{c}
x+a_{r}-(r-1) \\
a_{r}
\end{array}\right)
$$

where $a_{1} \geq a_{2} \geq \cdots \geq a_{r} \geq 0$. Moreover, if $\mathscr{I}$ is the ideal sheaf of $I$ then $\mathscr{J}$ is r-regular.

We apply this result in the following proposition.

Proposition 1.6. Let $I$ be a saturated ideal in $R=k\left[X_{0}, \ldots, X_{n}\right]$ and suppose that $L$ is a linear form which is not a zero divisor on $A=R / I$. Denote by $J$ the ideal $I / L I \simeq(I, L) /(L)$ in the polynomial ring $S=R / L$ and suppose that the Hilbert function of $S / J$ has maximal growth in degree $d$.

Let $\bar{I}=\left\langle I_{\leq d}\right\rangle$ and let $\overline{\mathscr{I}}$ be the sheafification of $\bar{I}$. Then $\overline{\mathscr{I}}$ is d-regular. Proof. We need to show that $h^{i}(\overline{\mathscr{I}}(t-i))=0$ for all $t \geq d$. We begin by showing that this is true for $i=1$. 
First note that from Lemma 1.4 we have that $\bar{I}$ is a saturated ideal. Thus (for a general linear form $L$ ) we can also assume that $L$ is not a zero divisor on the ring $R / \bar{I}$. Let $\bar{J}$ be the ideal $\bar{I} / L \bar{I} \simeq(\bar{I}, L) /(L)$ in the ring $S$.

By definition, $\bar{J}$ and $J$ both agree in degrees $\leq d$ since that is the case for $I$ and $\bar{I}$. Since $\bar{I}$ is generated in degree $\leq d$ and $\bar{J}$ is a quotient of $\bar{I}$ we have that $\bar{J}=\left\langle\bar{J}_{\leq d}\right\rangle=\left\langle J_{\leq d}\right\rangle$.

Note also that since $S / J$ has maximal growth in degree $d$ we have $S_{1} J_{d}=$ $J_{d+1}$. So, $J_{d+1}=\bar{J}_{d+1}$ also.

Claim. $(\bar{J})_{t}=\left((\bar{J})^{\text {sat }}\right)_{t}$ for all $t \geq d$.

Proof. $\bar{J}$ certainly agrees with its saturation for all $t$ sufficiently large. So, for definiteness, assume that this happens for all $t \geq N$. Then we have

$$
\begin{aligned}
& J_{d}=\bar{J}_{d} \subseteq\left((\bar{J})^{\text {sat }}\right)_{d} \subseteq S_{d} \\
& J_{d+1}=\bar{J}_{d+1} \subseteq\left((\bar{J})^{\text {sat }}\right)_{d+1} \subseteq S_{d+1} \\
& \begin{array}{cc}
\cdots & \cdots \\
\cdots & \cdots \\
\bar{J}_{N} & =\left((\bar{J})^{\text {sat }}\right)_{N}
\end{array} \\
& \ldots \quad \ldots
\end{aligned}
$$

Now $S / J$ has maximal growth in degree $d$, so, by construction, $S / \bar{J}$ has maximal growth in degree $\geq d$ thanks to the Gotzmann Persistence Theorem.

But, if for some $i, d<i<N$ we have

$$
\bar{J}_{i} \subset\left((\bar{J})^{\text {sat }}\right)_{i}
$$

then the Hilbert function of $S /\left(\bar{J}^{\text {sat }}\right)$ would fall behind that of $S / \bar{J}$. Since the latter Hilbert function is growing maximally the former can never catch up, although it does in degree $\geq N$. That contradiction establishes the claim.

Let $\overline{\mathscr{T}}$ be the sheafification of the ideal $\bar{J}$. If we shift the exact sequence of sheaves

$$
0 \rightarrow \overline{\mathscr{I}}(-1) \rightarrow \overline{\mathscr{I}} \rightarrow \overline{\mathscr{I}} \rightarrow 0
$$

by $t$ and take cohomology we have

$$
0 \rightarrow \bar{I}_{t-1} \rightarrow \bar{I}_{t} \rightarrow\left((\bar{J})^{\text {sat }}\right)_{t} \rightarrow H^{1}(\overline{\mathscr{I}})(t-1) \rightarrow H^{1}(\overline{\mathscr{I}})(t) .
$$

For $t \geq d$ the map

$$
\bar{I}_{t} \rightarrow\left((\bar{J})^{\text {sat }}\right)_{t}
$$

is, in view of the claim above, a surjection. Hence, the map

$$
H^{1}(\overline{\mathscr{I}})(t-1) \rightarrow H^{1}(\overline{\mathscr{I}})(t)
$$

is an inclusion for all $t \geq d$. Since, for $t \gg 0$ we must have $H^{1}(\overline{\mathscr{I}})(t)=0$ this completes the proof for $i=1$.

To prove the required vanishings of the higher cohomology groups of $\overline{\mathscr{I}}$ we pass to the ideal $\bar{J}=\left\langle J_{\leq d}\right\rangle$.

Suppose that $H(S / \bar{J}, d)=b_{d}$ and write the $d$-binomial expansion of $b_{d}$ as

$$
b_{d}=\left(\begin{array}{c}
m_{d} \\
d
\end{array}\right)+\left(\begin{array}{c}
m_{d-1} \\
d-1
\end{array}\right)+\cdots+\left(\begin{array}{c}
m_{j} \\
j
\end{array}\right), \quad j=d-r .
$$


From Gotzmann's Persistence and Regularity Theorems we can conclude that $\overline{\mathscr{T}}$ is $r$-regular (see 0.5 for the appropriate expression for the Hilbert function for $\bar{J}$ ). Since $r \leq d$ we conclude that $\overline{\mathscr{T}}$ is also $d$-regular.

We now show that $h^{i}(\mathscr{I}(t-i))=0$ for all $t \geq d$ and $i>1$. As before, we consider the exact sequence of sheaves

$$
0 \rightarrow \overline{\mathscr{I}}(-1) \rightarrow \overline{\mathscr{I}} \rightarrow \overline{\mathscr{T}} \rightarrow 0 .
$$

If we twist the exact sequence above by $t-i+1$ and take cohomology we get

$$
H^{i-1}(\overline{\mathscr{T}}(t-i+1)) \rightarrow H^{i}(\overline{\mathscr{I}}(t-i)) \rightarrow H^{i}(\overline{\mathscr{I}}(t-i+1)) .
$$

Since $\overline{\mathscr{T}}$ is $d$-regular we obtain $H^{i-1}(\overline{\mathscr{T}}(t-i+1))=0$ for all $t \geq d$. Thus, we have an inclusion $H^{i}(\overline{\mathscr{I}}(t-i)) \rightarrow H^{i}(\overline{\mathscr{I}}(t-i+1))$ for all $t \geq d$. Since, for all $s \gg 0$ we have $H^{i}(\mathscr{I}(s))=0$, that completes the proof of the proposition.

Remark 1.7. Notice that $\bar{J}=\bar{I} / L \bar{I}$ and that $L$ is not a zero divisor modulo $\bar{I}$. Nevertheless, we cannot conclude (and it is not, in general, the case) that $\bar{J}$ is a saturated ideal. Thus, it is noteworthy that with the maximal growth assumption we can conclude a regularity result about $\bar{I}$ from a regularity result about the saturation of $\bar{J}$. This in spite of the fact that we have only very partial information about the Hilbert function of $\bar{I}$.

However, the key fact here is that $\bar{J}$ agrees with its saturation in all degrees $\geq d$. This was at the heart of the results above. If we know this fact, for reasons other than maximal growth, then similar conclusions can be drawn. This idea is applied in Corollary 3.7.

\section{THE GREATEST COMMON DIVISOR OF A COMPONENT OF A HOMOGENEOUS IDEAL}

In this section we will generalize some results of E. Davis [D]. Our goal is to describe what happens when a homogeneous component of the saturated ideal of a scheme has a greatest common divisor (GCD), and especially to understand this in the case where there is maximal growth of the Hilbert function or at least when the Hilbert function has a certain special value. We will be especially interested in the case of zeroschemes in projective space. (Davis' situation was for codimension two Cohen-Macaulay subschemes in $\mathbb{P}^{n}$.)

We first define a collection of functions on the nonnegative integers, whose values are again nonnegative integers. For $r=2$ these functions were also studied by Raciti [Ra].

Definition 2.1. For $r \geq 1, k \geq 1$, and $x \geq k, f_{r, k}(x)=\left(\begin{array}{c}x+r \\ r\end{array}\right)-\left(\begin{array}{c}x-k+r \\ r\end{array}\right)$. Also, $f_{r, 0}(x)=0$ for all $r$ and all $x$. For $x \geq k$ this is the Hilbert polynomial of a degree $k$ hypersurface in $\mathbb{P}^{r}$.

Remark 2.2. We observe that for any choice of $k_{1}<k_{2} \leq x, f_{r, k_{1}}(x)<$ $f_{r, k_{2}}(x)$. In particular, $f_{r, k}(x)>0$ for all $r \geq 1, k \geq 1$, and $x \geq k$ as noted above.

Proposition 2.3. Let $I_{Z} \subset R=k\left[X_{0}, \ldots, X_{r+1}\right]$ be the saturated ideal of some scheme $Z$ (with no assumption on the dimension) and assume that $\left(I_{Z}\right)_{d}$ has a $G C D, F$, of degree $k$. Let $Z_{1}$ be the subscheme defined by $I_{Z_{1}}=\left[I_{Z}+(F)\right]^{\text {sat }}$ and $Z_{2}$ the subscheme defined by $\left[I_{Z}: F\right]$. Then 
(a) For any $i \leq d,\left[I_{Z}+(F)\right]_{i}=(F)_{i}$. However, it is not necessarily equal to $\left[I_{Z_{i}}\right]_{i}$.

(b) $\left[I_{Z}: F\right]=I_{Z_{2}}$.

(c) For $t \leq d, H\left(Z_{2}, t-k\right)=H(Z, t)-f_{r+1, k}(t)$.

(d) If $Z$ is reduced then so is $F$.

Proof. (d) is clear. (a) follows from the fact that $F$ is a factor of every element of $\left(I_{Z}\right)_{i}$ for $i \leq d$. The fact that $\left[I_{Z}+(F)\right]_{i}$ is not necessarily equal to $\left[I_{Z_{i}}\right]_{i}$ is illustrated in Example 2.11. (b) says simply that $\left[I_{Z}: F\right]$ is saturated. This is an easy exercise-it follows from the fact that $I_{Z}$ itself is saturated.

For $(\mathrm{c})$, observe that

$$
\frac{I_{Z}}{F \cdot\left[I_{Z}: F\right]}=\frac{I_{Z}}{(F) \cap I_{Z}} \cong \frac{I_{Z}+(F)}{(F)} .
$$

We thus have an exact sequence of graded modules

$$
0 \rightarrow\left[I_{Z}: F\right](-k) \stackrel{\times F}{\rightarrow} I_{Z} \rightarrow \frac{I_{Z}+(F)}{(F)} \rightarrow 0,
$$

and combining this with the fact that $\left[I_{Z}: F\right]=I_{Z_{2}}$ we get

$$
0 \rightarrow I_{Z_{2}}(-k) \stackrel{\times F}{\rightarrow} I_{Z} \stackrel{\phi}{\rightarrow} \frac{I_{Z}+(F)}{(F)} \rightarrow 0 .
$$

The last term is zero in degree $\leq d$ by (a), so

$$
\operatorname{dim}\left(I_{Z_{2}}\right)_{t-k}=\operatorname{dim}\left(I_{Z}\right)_{t}
$$

for all $t \leq d$. We rewrite this as

$$
\begin{aligned}
& \left(\begin{array}{c}
t-k+r+1 \\
t-k
\end{array}\right)-\operatorname{dim}\left(I_{Z_{2}}\right)_{t-k} \\
& \quad=\left(\begin{array}{c}
t+r+1 \\
t
\end{array}\right)-\operatorname{dim}\left(I_{Z}\right)_{t}+\left(\begin{array}{c}
t-k+r+1 \\
t-k
\end{array}\right)-\left(\begin{array}{c}
t+r+1 \\
t
\end{array}\right)
\end{aligned}
$$

for $t \leq d$. That is,

$$
H\left(Z_{2}, t-k\right)=H(Z, t)-\left[\left(\begin{array}{c}
t+r+1 \\
t
\end{array}\right)-\left(\begin{array}{c}
t-k+r+1 \\
t-k
\end{array}\right)\right]
$$

for all $t \leq d$, as claimed.

Theorem 2.4. Let $I_{Z} \subset R=k\left[X_{0}, \ldots, X_{r+1}\right]$ be the saturated ideal of some scheme $Z$ (with no assumption on the dimension) and assume that $\left(I_{Z}\right)_{d}$ has a GCD, $F$, of degree $k$. Assume further that $\Delta H(Z, d)=f_{r, k}(d)$. Then

(a) $I_{Z}+(F)=I_{Z_{1}}$ and $\left[I_{Z}: F\right]=I_{Z_{2}}$.

$$
\Delta H\left(Z_{2}, t-k\right)= \begin{cases}\Delta H(Z, t)-f_{r, k}(t), & \text { for } t \leq d ; \\ 0, & \text { for } t \geq d .\end{cases}
$$

In particular, $\operatorname{dim} Z_{2}=0$.

(c)

$$
\Delta H\left(Z_{1}, t\right)= \begin{cases}f_{r, k}(t), & \text { for } t \leq d \\ \Delta H(Z, t), & \text { for } t \geq d\end{cases}
$$


(d) If $Z$ is reduced then so is $F$.

Proof. We have already seen (d) and the second half of (a). (b) follows immediately from Proposition 2.3 since then $\Delta H\left(Z_{2}, d-k\right)=0$ and so $\Delta H\left(Z_{2}, t\right)=0$ for all $t \geq d-k$. This says that $Z_{2}$ is a zeroscheme, and hence it follows from $\Delta H\left(Z_{2}, \bar{d}-k\right)=0$ that $h^{1}\left(\mathscr{I}_{Z_{2}}(t)\right)=0$ for all $t \geq d-k-1$. Now, we have an exact sequence of modules

$$
0 \rightarrow I_{Z} \cap(F) \rightarrow I_{Z} \oplus(F) \rightarrow I_{Z}+(F) \rightarrow 0
$$

and since $F \cdot I_{Z_{2}}=F \cdot\left[I_{Z}: F\right]=I_{Z} \cap(F)$ this gives

$$
0 \rightarrow I_{Z_{2}}(-k) \stackrel{\times F}{\rightarrow} I_{Z} \oplus(F) \rightarrow I_{Z}+(F) \rightarrow 0 .
$$

For $t \geq d-1$ we have seen that $h^{1}\left(\mathscr{I}_{Z_{2}}(t-k)\right)=0$, so we can sheafify the above sequence, twist by $t \geq d-1$ and take cohomology to get a short exact sequence

$$
\left.0 \rightarrow I_{Z_{2}}(-k)_{t} \rightarrow\left[I_{Z} \oplus(F)\right]_{t} \rightarrow\left[H_{*}^{0}\left(I_{Z} \widetilde{+(F}\right)\right)\right]_{t} \rightarrow 0 .
$$

(The rightmost term is just the degree $t$ component of $I_{Z_{1}}$.) In particular, comparing this sequence with the one preceding it, we get that $I_{Z}+(F)$ agrees with $I_{Z_{1}}$ in degrees $\geq d-1$. In particular,

$$
h^{0}\left(\mathscr{I}_{Z_{1}}(t)\right)=\left(\begin{array}{c}
t-k+r+1 \\
t-k
\end{array}\right)+h^{0}\left(\mathscr{I}_{Z}(t)\right)-h^{0}\left(\mathscr{I}_{Z_{2}}(t-k)\right) \quad \text { for } t \geq d-1 .
$$

Subtracting $\left(\begin{array}{c}t+r+1 \\ t\end{array}\right)$ from both sides and simplifying gives

$$
H\left(Z_{1}, t\right)=H(Z, t)-H\left(Z_{2}, t-k\right) \text { for } t \geq d-1 .
$$

Then taking first difference gives that for $t \geq d$ we have

$$
\Delta H\left(Z_{1}, t\right)=\Delta H(Z, t)-\Delta H\left(Z_{2}, t-k\right)=\Delta H(Z, t) .
$$

In particular,

$$
\Delta H\left(Z_{1}, d\right)=\left(\begin{array}{c}
d+r \\
r
\end{array}\right)-\left(\begin{array}{c}
d-k+r \\
d-k
\end{array}\right)=f_{r, k}(d)
$$

We want to show that $I_{Z_{1}}=I_{Z}+(F)$, and we have seen above that it is true in degree $\geq d-1$. Furthermore we have seen that in degree $\leq d$ we have $I_{Z}+(F)=(F)$. What we claim is that in fact $I_{Z_{1}}=(F)=\left[I_{Z}+(F)\right]$ in degree $\leq d$. This will prove the remaining parts of (a) and (c), and complete the proof of the theorem.

Certainly we have an inclusion $(F) \subseteq I_{Z_{1}}$ in all degrees, and we have just seen that they are equal in degrees $d-1$ and $d$. Suppose that they are not equal for some degree $i \leq d-2$. This means that there is some element $G \in\left(I_{Z_{1}}\right)_{i}$ which does not have $F$ as a factor. But then for a general linear form $L, G \cdot L^{d-i}$ is an element of $\left(I_{Z_{1}}\right)_{d}$ which does not have $F$ as a factor. Contradiction.

Our next results illustrate again the power of maximal growth of the Hilbert function: it can force the existence of a GCD and determine its degree. (And then the above results apply.) We first make an observation which will be useful for much of the rest of the section. (In the case $r=2$ it was essentially observed by Green in [Gr], immediately before Theorem 4.) 
Lemma 2.5. Let $d \geq k>0$. Then

$$
\begin{aligned}
f_{r, k}(d) & =\left(\begin{array}{c}
d+r \\
d
\end{array}\right)-\left(\begin{array}{c}
d-k+r \\
d-k
\end{array}\right) \\
& =\left(\begin{array}{c}
d+r-1 \\
d
\end{array}\right)+\left(\begin{array}{c}
d+r-2 \\
d-1
\end{array}\right)+\cdots+\left(\begin{array}{c}
d+r-k \\
d-k+1
\end{array}\right) \\
& =\left(\begin{array}{c}
d+r-1 \\
r-1
\end{array}\right)+\left(\begin{array}{c}
d+r-2 \\
r-1
\end{array}\right)+\cdots+\left(\begin{array}{c}
d+r-k \\
r-1
\end{array}\right) .
\end{aligned}
$$

Proof. For convenience let $A=\left(\begin{array}{c}d+r \\ d\end{array}\right), B=\left(\begin{array}{c}d-k+r \\ d-k\end{array}\right)$, and $C_{i}=\left(\begin{array}{c}d+r-i \\ d-i+1\end{array}\right)$ (for $1 \leq i \leq k)$. We first want to show that $B=A-C_{1}-C_{2}-\cdots-C_{k}$. Just observe that $A-C_{1}=\left(\begin{array}{c}d+r-1 \\ d-1\end{array}\right), A-C_{1}-C_{2}=\left(\begin{array}{c}d+r-2 \\ d-2\end{array}\right)$, etc. The second part of the lemma follows immediately from the first.

Definition 2.6. Let $I \subset S=k\left[X_{0}, \ldots, X_{r}\right]$ be a homogeneous ideal. Assume that $I_{d} \neq 0$, so $H(S / I, d)<\left(\begin{array}{c}d+r \\ r\end{array}\right)$. The potential $G C D$ of $I_{d}$ is

$$
\max \left\{k \mid f_{r, k}(d) \leq H(S / I, d)\right\} \text {. }
$$

(Note that this makes sense because of Remark 2.2 and the definition of $f_{r, k}$.)

Proposition 2.7. Let $I \subset S=k\left[X_{0}, \ldots, X_{r}\right]$ be an arbitrary homogeneous ideal and let $d$ be such that $I_{d} \neq 0$. Assume that $0<k=$ potential $G C D$ of $I_{d}$. Assume further that $S / I$ has maximal growth in degree $d$. Then both $I_{d}$ and $I_{d+1}$ have a GCD, $F$, of degree $k$.

Proof. We know that $H(S / I, d)<\left(\begin{array}{c}d+r \\ r\end{array}\right)-\left(\begin{array}{c}d-(k+1)+r \\ r\end{array}\right)=f_{r, k+1}(d)$. (If $d>k$ this is by definition of potential GCD; if $d=k$ then $f_{r, k+1}(d)$ is not defined, but this inequality follows from the assumption that $I_{d} \neq 0$.)

By Lemma 2.5, the first $k$ terms of $f_{r, k}(d)$ and $f_{r, k+1}(d)$ are equal. Since $f_{r, k}(d) \leq H(S / I, d)<f_{r, k+1}(d)$, we get

$$
\begin{aligned}
H(S / I, d)= & \left(\begin{array}{c}
d+r-1 \\
d
\end{array}\right)+\left(\begin{array}{c}
d+r-2 \\
d-1
\end{array}\right)+\cdots+\left(\begin{array}{c}
d+r-k \\
d-k+1
\end{array}\right) \\
& +\left(\begin{array}{c}
c \\
d-k
\end{array}\right)+\text { (lower terms). }
\end{aligned}
$$

Observe that $c<d+r-(k+1)$ since $H(S / I, d)<\left(\begin{array}{c}d+r \\ r\end{array}\right)-\left(\begin{array}{c}d-(k+1)+r \\ r\end{array}\right)$ (use Lemma 2.5 again). So

$$
\begin{aligned}
H(S / I, d)= & \left(\begin{array}{c}
d+r-1 \\
r-1
\end{array}\right)+\left(\begin{array}{c}
d+r-2 \\
r-1
\end{array}\right)+\cdots+\left(\begin{array}{c}
d+r-k \\
r-1
\end{array}\right) \\
& +\left(\operatorname{terms}\left(\begin{array}{c}
c \\
i
\end{array}\right) \text { with } i<r-1\right) .
\end{aligned}
$$

Let $V$ be the scheme defined by $\left\langle I_{\leq d}\right\rangle$. As in Lemma 1.4, let $\bar{I}=\left\langle I_{\leq d}\right\rangle=I_{V}$. By the Gotzmann Persistence Theorem,

$$
H(S / \bar{I}, t)=\left(\begin{array}{c}
t+r-1 \\
r-1
\end{array}\right)+\left(\begin{array}{c}
t+r-2 \\
r-1
\end{array}\right)+\cdots+\left(\begin{array}{c}
t+r-k \\
r-1
\end{array}\right)+(\text { lower terms })
$$

is the Hilbert polynomial of $S / I_{V}$. It follows that $\operatorname{dim} V=r-1$ and $\operatorname{deg} V=k$ as claimed. 
Interestingly, if the values of both $H(S / I, d)$ and $H(S / I, d+1)$ fall exactly on one of the curves $f_{r, k}(x)$ then we can say more:

Corollary 2.8. Let $I \subset S$ be an ideal, $S=k\left[X_{0}, \ldots, X_{r}\right]$. Assume that

$$
\begin{aligned}
H(S / I, d) & =\left(\begin{array}{c}
d+r \\
d
\end{array}\right)-\left(\begin{array}{c}
d-k+r \\
d-k
\end{array}\right), \\
H(S / I, d+1) & =\left(\begin{array}{c}
d+1+r \\
d+1
\end{array}\right)-\left(\begin{array}{c}
d+1-k+r \\
d+1-k
\end{array}\right),
\end{aligned}
$$

where $d \geq k$. Then $I_{d}=(F)_{d}$ and $I_{d+1}=(F)_{d+1}$ for some $F \in S_{k}$.

Proof. By Lemma 2.5,

$$
\begin{aligned}
H(S / I, d) & =\left(\begin{array}{c}
d+r-1 \\
d
\end{array}\right)+\left(\begin{array}{c}
d+r-2 \\
d-1
\end{array}\right)+\cdots+\left(\begin{array}{c}
d+r-k \\
d-k+1
\end{array}\right), \\
H(S / I, d+1) & =\left(\begin{array}{c}
d+r \\
d+1
\end{array}\right)+\left(\begin{array}{c}
d+r-1 \\
d
\end{array}\right)+\cdots+\left(\begin{array}{c}
d+1+r-k \\
d-k+2
\end{array}\right) .
\end{aligned}
$$

These are the $d$-binomial expansion of $(S / I, d)$ and the $(d+1)$-binomial expansion of $H(S / I, d+1)$, respectively, and by Macaulay's Theorem the value in degree $d+1$ represents the maximum possible growth of the Hilbert function given the value in degree $d$.

As above, let $V$ be the scheme defined by the saturated ideal (Lemma 1.4) $\bar{I}=\left\langle I_{\leq d}\right\rangle$ (or, equivalently, by $\left\langle I_{\leq d+1}\right\rangle$ since maximal growth in degree $d$ for an ideal $I$ implies that $I$ does not have a minimal generator in degree $d+1$ ). We have seen that $V$ has dimension $r-1$ and degree $k$, but we have to eliminate the possibility of higher codimensional components. Now we get that the Hilbert polynomial of $S / I_{V}$ is precisely

$$
H\left(S / I_{V}, t\right)=\left(\begin{array}{c}
t+r-1 \\
r-1
\end{array}\right)+\left(\begin{array}{c}
t+r-2 \\
r-1
\end{array}\right)+\cdots+\left(\begin{array}{c}
t+r-k \\
r-1
\end{array}\right)
$$

by the Gotzmann Persistence Theorem. Let $F$ be a (not necessarily irreducible) homogeneous polynomial of degree $k$ defining the dimension $r-1$ part of $V$. We have that $I_{V} \subseteq(F)$. In particular, $I_{d}=\left(I_{V}\right)_{d} \subseteq(F)_{d}$. But since $H(S /(F), d)=\left(\begin{array}{c}d+r \\ d\end{array}\right)-\left(\begin{array}{c}d-k+r \\ d-k\end{array}\right)$ (and similarly for $H(S /(F), d+1)$ ) we get equalities: $I_{d}=\left(I_{V}\right)_{d}=(F)_{d}$ and $I_{d+1}=\left(I_{V}\right)_{d+1}=(F)_{d+1}$. (The fact that $I_{V}$ is generated in degree $\leq k$ also follows easily from Gotzmann's Regularity Theorem, once we know the Hilbert polynomial.)

Corollary 2.9. Let $I_{Z}$ be the saturated ideal of a closed subscheme $Z$ of $\mathbb{P}^{r+1}$. Assume that $\triangle H(Z, d)$ has potential $G C D=k \geq 1$ and maximal growth in degree $d$. Then the elements of $\left(I_{Z}\right)_{d}$ and $\left(I_{Z}\right)_{d+1}$ have a GCD of degree $k$. (Hence Proposition 2.3 applies.)

Proof. As usual we let $J$ denote the ideal $\left(I_{z}+(L)\right) /(L)$ where $L$ is a general linear form. By Proposition 2.7, $J$ has a GCD of degree $k$ in degrees $d$ and $d+1$; hence the same holds for $I_{Z}$.

As before, if $\Delta H(Z, d)=f_{r, k}(d)$ and it is known that there is a GCD, or in particular if $\Delta H(Z, d)$ and $\Delta H(Z, d+1)$ both lie on the curve $f_{r, k}(x)$ then we can say more: 
Corollary 2.10. If $\Delta H(Z, d)=\left(\begin{array}{c}d+r \\ d\end{array}\right)-\left(\begin{array}{c}d-k+r \\ d-k\end{array}\right)$ and $\Delta H(Z, d+1)=\left(\begin{array}{c}d+1+r \\ d+1\end{array}\right)-$ $\left(\begin{array}{c}d+1-k+r \\ d+1-k\end{array}\right)$ then $\left(I_{Z}\right)_{d}$ and $\left(I_{Z}\right)_{d+1}$ have a GCD of degree $k$. Hence Theorem 2.4 applies and the Hilbert functions of both $Z_{1}$ and $Z_{2}$ are completely determined.

Proof. Immediate from Corollary 2.8.

These results are related to work of Davis in the following sense. In [D] Theorem 4.1, Davis considers arithmetically Cohen-Macaulay codimension two schemes $Z$ and the Hilbert function of the Artinian reduction of the coordinate ring of $Z$. If a component of the ideal of $Z$ has a GCD of degree $d$ and if the value of the Hilbert function of the Artinian reduction is also $d$ then he reaches a conclusion similar to that in our Theorem 2.4.

In the case of points in $\mathbb{P}^{2}$, this result is exactly ours. In higher dimension and/or codimension, however, we make the following observations. First, we do not assume that $Z$ is arithmetically Cohen-Macaulay or of codimension two. (In private conversation with the second author he claims that the codimension two assumption is not necessary for his results, properly reformulated. However, he asserts that arithmetically Cohen-Macauly is necessary.)

Second, even if $Z$ is arithmetically Cohen-Macaulay and has codimension two, the hypotheses of the theorems are still different. Davis passes to the Artinian reduction (i.e., considering restriction to a general line) while we consider the first difference of the Hilbert function (i.e., restricting to a general hyperplane).

For the remainder of this section, $Z \subset \mathbb{P}^{3}$ will be a zeroscheme with saturated ideal $I_{Z} \subset R=k\left[X_{0}, \ldots, X_{3}\right]$. Notice that if $Z$ is reduced and $\left(I_{Z}\right)_{d}$ has a GCD, and if $Z_{1}$ and $Z_{2}$ are as defined in Proposition 2.3, then $Z_{1}$ is the subset of points lying on the GCD and $Z_{2}$ is the subset lying off the GCD, and $Z=$ $Z_{1} \cup Z_{2}$. Let $L$ be a general linear form; without loss of generality set $L=X_{3}$. Let $S=k\left[X_{0}, X_{1}, X_{2}\right]=R /(L)$. Let $J=I_{Z} /\left(L \cdot I_{Z}\right)=\left(I_{Z}+(L)\right) /(L) \subset S$. Let $H(Z,-)$ be the Hilbert function of $R / I_{Z}$. Since $Z$ is arithmetically CohenMacaulay, the Hilbert function of $S / J$ is exactly $\Delta H(Z,-)$ and it makes sense to ask if this Hilbert function has maximal growth in a given degree $d$. (See 1.1 for the relation between maximal growth for $R / I_{Z}$ and for $S / J$ in a given degree.)

Example 2.11. Let $Z \subset \mathbb{P}^{3}$ be a set of points whose Hilbert function has first difference

$$
\begin{array}{lllll}
1 & 3 & 4 & 5 & 0 .
\end{array}
$$

Here the potential GCD of $\left(I_{Z}\right)_{2}$ is $k=1$ and the growth in degree 2 is maximal, so by Proposition 2.7, $\left(I_{Z}\right)_{2}$ and $\left(I_{Z}\right)_{3}$ have a GCD of degree 1 . Let $Z_{1}$ be the subset on this plane and $Z_{2}$ the subset off the plane. Then by Proposition 2.3 (c) (taking the first difference of that statement and applying it to $d=3$ ), the Hilbert function of $Z_{2}$ can be computed by

$$
\begin{array}{rrrr}
t: 0 & 1 & 2 & 3 \\
\Delta H(Z, t): 1 & 3 & 4 & 5 \\
\Delta f_{3,1}(t): 1 & 2 & 3 & 4 \\
\Delta H\left(Z_{2},-\right): 0 & 1 & 1 & 1
\end{array}
$$


That is, $Z_{2}$ consists of at least 3 points on a line. It cannot have more than 4 points on a line since the Hilbert function of $Z$ does not allow more than 4 points on a line. Thus $Z_{1}$ consists of either 9 or 10 points on a plane. Both are possible. Each of the following examples gives this Hilbert function:

Case 1. Choose a line $\lambda$ and a plane $\Lambda$ not containing $\lambda$. Let $Z_{1}$ be a general set of 10 points on $\Lambda$ and $Z_{2}$ a general set of 3 points on $\lambda$. Any cubic surface containing $Z$ and not containing $\Lambda$ as a component would restrict to a cubic curve in $\Delta$ containing $Z_{1}$.

Case 2. Choose a line $\lambda$ and a plane $\Lambda$ not containing $\lambda$. Let $P$ be the intersection point of $\Lambda$ and $\lambda$. Let $Z_{1}$ be a general set of 9 points on $\Lambda$ and $Z_{2}$ a general set of 4 points on $\lambda$. Any cubic surface containing $Z$ must contain $\lambda$ and hence $P$. So it restricts to a cubic curve in $\Lambda$ containing the 10 points $Z_{1} \cup\{P\}$.

This illustrates the fact that one cannot completely specify the Hilbert functions of $Z_{1}$ and $Z_{2}$ given only the hypotheses of Proposition 2.3. Thus a result like Theorem 2.4 requires the extra assumption.

Case 2 also illustrates the remark in Proposition 2.3 that $\left[I_{Z}+(F)\right]_{i}=(F)_{i}$ is not necessarily equal to $\left[I_{Z_{i}}\right]_{i}$ for $i \leq d$. Indeed, in our situation, for $i=3$ the former has dimension 10 while the latter has dimension 11.

We remark that, from the techniques we have developed, one can pick up the line $\lambda$ in a slightly different way. Let $\bar{I}=\left(I_{Z}\right)_{\leq 3}$ be the saturated (by Lemma 1.4) ideal of a scheme $V$. Then the Hilbert polynomial of the general hyperplane section of $V$ is $\left(\begin{array}{c}x+1 \\ 1\end{array}\right)+1$, so $V$ consists of a plane, a line plus possibly finitely many points. (But our analysis above rules out the extra points.)

Example 2.12. We would like to describe the functions $f_{r, k}$ in $\mathbb{P}^{3}$. In this case $r=2$ (since we are always looking at the quotient ring $S$ ) and one can check that

$$
f_{2, k}(x)=k x-\frac{k^{2}-3 k}{2}=k x-\left(\begin{array}{c}
k-1 \\
2
\end{array}\right)+1
$$

for $x \geq k$. (This is the Hilbert polynomial of a plane curve of degree $k$, as one would expect.) These are exactly the equations of the lines studied by Raciti in [Ra]. These lines (if we extend them to the left) are all tangent to the parabola $y=\frac{1}{2} x^{2}+\frac{3}{2} x+\frac{9}{8}$.

\section{Zeroschemes}

In this section $Z$ will denote a finite set of points in $\mathbb{P}^{r+1}$ with saturated ideal $I_{Z} \subset R=k\left[X_{0}, \ldots, X_{r+1}\right]$. Let $L$ be a general linear form; without loss of generality set $L=X_{r+1}$. Let $S=k\left[X_{0}, \ldots, X_{r}\right]=R /(L)$. Let $J=$ $I_{Z} /\left(L \cdot I_{Z}\right)=\left(I_{Z}+(L)\right) /(L) \subset S$. Let $H(Z,-)$ be the Hilbert function of $R / I_{Z}$. Since $Z$ is arithmetically Cohen-Macaulay, the Hilbert function of $S / J$ is exactly $\Delta H(Z,-)$, and it makes sense to ask if this latter Hilbert function has maximal growth in a given degree $d$. (See Lemma 1.1 for the relation between maximal growth for $R / I_{Z}$ and for $S / J$ in a given degree.)

In $\S 2$ we saw how certain kinds of maximal growth of the Hilbert function $\Delta H(Z,-)$ can force a large subset $Z_{1}$ of $Z$ to lie on a hypersurface; in fact, one can often say exactly how large $Z_{1}$ is. In many cases one can also say quite 
a bit about the points $Z_{2}$ of $Z$ off the hypersurface, sometimes getting all the values of the Hilbert function of that set as well.

Our object in this section is to show how maximal growth of the Hilbert function can also be used to force many points of $Z$ to lie on a recognizable subvariety of higher codimension, and again allow us to say how many such points there are (or at least give a very strong lower bound). We will improve these results even further in the next section when we assume some uniformity about the points of $Z$.

We start with a result which can be shown to be equivalent to Theorem 3 of [Gr]. However, we state it in the context of maximal growth of the Hilbert function, and our proof is completely different from that of [Gr].

Lemma 3.1. Let $I \subset S$ be an ideal satisfying $H(S / I, d)=\left(\begin{array}{c}d+m \\ d\end{array}\right)$ and $H(S / I, d+1)=\left(\begin{array}{c}d+1+m \\ d+1\end{array}\right)$. Then $I_{d}$ is the degree $d$ component of the saturated ideal of an $m$-dimensional linear space in $\mathbb{P}^{r}$ (and similarly for $I_{d+1}$ ).

Proof. The given values of the Hilbert function represent maximal growth in degree $d$. Let $V$ be the scheme defined by the saturated (Lemma 1.4) ideal $\bar{I}=$ $\left\langle I_{\leq d}\right\rangle$. The Hilbert polynomial of $V$ is $\left(\begin{array}{c}t+m \\ t\end{array}\right)$ so $\operatorname{deg} V=1$ and $\operatorname{dim} V=m$. As in Corollary 2.8 , we have to make sure there are no higher codimensional components. Let $\Lambda$ be the dimension $m$ component. If $V$ has any higher codimensional component then $I_{V} \subset I_{\Lambda}$ (where this is a strict inclusion). Hence for $t \gg 0,\left(\begin{array}{c}t+m \\ t\end{array}\right)=H\left(S / I_{V}, t\right)>H\left(S / I_{\Lambda}, t\right)=\left(\begin{array}{c}t+m \\ t\end{array}\right)$. Contradication.

The same proof, combined with Corollary 2.8 , gives our analog of [Gr, Theorem 4], but stated more generally to allow higher dimension, and stated in terms of maximal growth of the Hilbert function. This will be used in Corollary 3.10.

Corollary 3.2. Let $I \subset S$ be an ideal satisfying $H(S / I, d)=\left(\begin{array}{c}d+m \\ d\end{array}\right)-\left(\begin{array}{c}d-k+m \\ d-k\end{array}\right)$ and $H(S / I, d+1)=\left(\begin{array}{c}d+1+m \\ d+1\end{array}\right)-\left(\begin{array}{c}d+1-k+m \\ d+1-k\end{array}\right)$, with $m \geq 2$. Then $I_{d}$ is the degree $d$ component of the saturated ideal of a hypersurface of degree $k$ inside a $\mathbb{P}^{m}$ of $\mathbb{P}^{r}$. That is, $I_{d}$ is the degree $d$ component of the saturated ideal of the complete intersection of a homogeneous polynomial of degree $k$ and $r-m$ linear forms.

Proof. The point is that, as in Corollary 2.8 , this is maximal growth. The Hilbert polynomial one then obtains is the smallest possible for a variety of dimension $m-1$ and degree $k$. (See Remark 0.6.)

Although we are interested in zeroschemes in this section, we prove the next theorem in greater generality to give an indication of how our techniques extend beyond points.

Theorem 3.3. Let $Y$ be a closed, reduced subscheme of $\mathbb{P}^{r+1}$ of dimension $\geq 0$, with the first difference of the Hilbert function satisfying $\Delta H(Y, d)=\left(\begin{array}{c}d+m-1 \\ d\end{array}\right)$ and $\Delta H(Y, d+1)=\left(\begin{array}{c}d+m \\ d+1\end{array}\right)$. Then:

(a) $Y$ is the disjoint union of a scheme $Y_{1}$ and a scheme $Y_{2}$, where $Y_{1}$ lies in $a \mathbb{P}^{m}=\Lambda$ and $Y_{2}$ is a finite set of points.

(b) The first difference of the Hilbert function of $Y_{1}$ is

$$
\Delta H\left(Y_{1}, t\right)= \begin{cases}\Delta H(\Lambda, t), & \text { if } t \leq d+1 \\ \Delta H(Y, t), & \text { if } t \geq d .\end{cases}
$$

(c) $\left(I_{Y_{1}}\right)_{t}=\left(I_{Y}+I_{\Lambda}\right)_{t}$ for all $t \geq d-1$. 
Proof. (Observe that in particular we are claiming that the values of $\Delta H(\Lambda, t)$ and $\Delta H(Y, t)$ agree in degrees $d$ and $d+1$.) Let $L$ be a general linear form and let $J=\left(I_{Y}+(L)\right) /(L)$. As usual, $J$ is an ideal in the quotient ring $S$ and $H(S / J, t)=\Delta H(Y, t)$ for all $t$. The condition on $\Delta H(Y, d)$ and $\Delta H(Y, d+1)$ is the maximal growth condition of Lemma 3.1 (with $m-1$ instead of $m$ ), so $J_{d}$ is the degree $d$ component of the ideal of an $(m-1)$-plane $\bar{\Lambda} \subset \mathbb{P}^{r}$. Hence $\left(I_{Y}\right)_{\leq d}$ defines a scheme $V$ with support consisting of an $m$-plane $\Lambda$ and a finite set of points. (If there were components of dimension $\geq 1$ other than $\Lambda$, they would be picked up in the general hyperplane section.)

Let $Y_{1}$ be the subscheme of $Y$ lying on $\Lambda$ and $Y_{2}$ the "residual" subscheme. This makes sense since $Y$ is reduced. Since $Y \subseteq V$, the observations in the previous paragraph imply that $Y_{2}$ is a finite set of points. This proves (a). Let $W$ be the subscheme of $V$ supported on $Y_{2}$ and $X$ the rest. Note that $W \cap X=\varnothing$ and $I_{W} \cap I_{X}=I_{V}$. We thus have an exact sequence of modules

$$
0 \rightarrow I_{V} \rightarrow I_{W} \oplus I_{X} \rightarrow I_{W}+I_{X} \rightarrow 0 .
$$

Sheafify and twist by $t \geq d-1$. When we take cohomology we get

$$
H^{1}\left(\mathscr{I}_{V}(t)\right) \rightarrow H^{1}\left(\mathscr{J}_{W}(t)\right) \oplus H^{1}\left(\mathscr{I}_{X}(t)\right) \rightarrow H^{1}\left(\mathscr{O}_{\mathbb{P}^{r+1}}(t)\right)
$$

so by Proposition 1.6 we get that $h^{1}\left(\mathscr{J}_{W}(t)\right)=0$ for all $t \geq d-1$. But $Y_{2} \subseteq W$ and both are zeroschemes, so we get $h^{1}\left(\mathscr{I}_{Y_{2}}(t)\right)=0$ for all $t \geq d-1$. Therefore $\Delta H\left(Y_{2}, t\right)=0$ for all $t \geq d$.

Now let $L$ be a linear form vanishing on $\Lambda$ but not on any point of $Y_{2}$. As in $\S 2$ we have $I_{Y} \cap(L)=\left[I_{Y}: L\right] \cdot L=I_{Y_{2}} \cdot L$. Hence we have an exact sequence

$$
0 \rightarrow I_{Y_{2}}(-1) \stackrel{\times L}{\rightarrow} I_{Y} \oplus(L) \rightarrow I_{Y}+(L) \rightarrow 0 .
$$

Sheafifying, twisting by $t \geq d$ and taking cohomology, we get

$$
0 \rightarrow\left(I_{Y_{2}}\right)_{t-1} \rightarrow\left(I_{Y}\right)_{t} \oplus(L)_{t} \rightarrow\left(I_{Z_{1}}\right)_{t} \rightarrow 0 .
$$

As in the proof of Theorem 2.4, this gives $H\left(Y_{1}, t\right)=H(Y, t)-H\left(Y_{2}, t-1\right)$ for all $t \geq d$. Thus $\Delta H\left(Y_{1}, t\right)=\Delta H(Y, t)-\Delta H\left(Y_{2}, t-1\right)$ for all $t \geq d+1$. We conclude that $\Delta H\left(Y_{1}, d+1\right)=\left(\begin{array}{c}d+m \\ m-1\end{array}\right)=\Delta H(Y, d+1)=\Delta H(\Lambda, d+1)$ and in fact $\Delta H\left(Y_{1}, t\right)=\Delta H(Y, t)$ for all $t \geq d+2$ as well. This proves the second half of $(b)$, and we now prove the first half.

We claim that $I_{Y_{1}}$ and $I_{\Lambda}$ agree in degree $\leq d+1$. Certainly $I_{\Lambda} \subseteq I_{Y_{1}}$, so $H\left(Y_{1}, t\right) \leq H(\Lambda, t)$ for all $t$. If $H\left(Y_{1}, t\right)<H(\Lambda, t)$ for any $t \leq d+1$, let $i$ be the minimum such. $i$ cannot be $d+1$ because otherwise it contradicts the fact that $\Delta H\left(Y_{1}, d+1\right)=\Delta H(\Lambda, d+1)$ (since by definition of $i$ the Hilbert functions agree in degree $<i)$. And if $i<d+1$ then the first difference of the Hilbert function of $Y_{1}$ can never catch up with that of $\Lambda$ since the latter has maximal growth for all degrees $\geq 1$. This proves the first half of $(b)$.

For the last part of the theorem, consider the exact sequence

$$
0 \rightarrow I_{Y} \cap I_{\Lambda} \rightarrow I_{Y} \oplus I_{\Lambda} \rightarrow I_{Y}+I_{\Lambda} \rightarrow 0 .
$$

Sheafify and twist by $d-1$. To prove the last part of the theorem, then, it is enough to show that $h^{1}\left(I_{Y} \cap I_{\Lambda}(d-1)\right)=h^{1}\left(\mathscr{I}_{\Lambda \cap Y_{2}}(d-1)\right)=0$. Consider the exact sequence

$$
0 \rightarrow \mathscr{I}_{V} \rightarrow \mathscr{I}_{\Lambda \cup Y_{2}} \rightarrow \mathscr{I}_{\Lambda \cup Y_{2}} / \mathscr{I}_{V} \rightarrow 0 .
$$


The last sheaf is supported on a zero-dimensional scheme, and we have already seen that $h^{1}\left(\mathscr{I}_{V}(d-1)\right)=0$. The result then follows.

Corollary 3.4. Let $Z \subset \mathbb{P}^{r+1}$ be a reduced set of points with $\Delta H(Z, d)=$ $\left({ }_{d}^{d+m-1}\right)$ and $\Delta H(Z, d+1)=\left(\begin{array}{c}d+m \\ d+1\end{array}\right)$. Then $Z$ contains a subset $Z_{1}$ lying on $a \mathbb{P}^{m}=\Lambda$, and the first difference of the Hilbert function of $Z_{1}$ is the sequence

$$
1 \quad m\left(\begin{array}{c}
m+1 \\
2
\end{array}\right) \cdots\left(\begin{array}{c}
d+m-1 \\
d
\end{array}\right)\left(\begin{array}{c}
d+m \\
d+1
\end{array}\right) \Delta H(Z, d+2) \cdots .
$$

(In particular, $Z$ contains at least $\left(\begin{array}{c}m+d+1 \\ m\end{array}\right)$ points on a $\mathbb{P}^{m}$.)

If we let $m=1, m$ as in Corollary 3.4, we obtain Proposition 5.2 of [GMR]. For $d$ as in the corollary and for $m=1$ the Hilbert function $\Delta H(Z, t)$ is completely determined for all $t \geq d$ by the cardinality of $Z$. This is not the case for $m>1$. Thus our description of the Hilbert function of $Z_{1}$ beyond the point of maximal growth represents a novel ingredient not present in the case $m=1$.

In $\S 2$ we generalized several theorems of Davis [D] by viewing those results as statements saying that maximal growth of a certain kind forces the existence of a GCD. For example, suppose the first difference of the Hilbert function of a set, $Z$, of points in $\mathbb{P}^{2}$ has flat growth from degree $d$ to $d+1$. Then this is maximal growth and hence it forces the components of the ideal in those two degrees to have a GCD. Furthermore, it forces a large subset $Z_{1}$ of $Z$ to lie on that GCD. Finally, the first difference of the Hilbert function of $Z_{1}$ agrees with that of the GCD up to degree $d+1$ and it agrees with that of $Z$ past $d+1$.

However, hypersurfaces in $\mathbb{P}^{2}$ are also curves, so Davis' results can be viewed as forcing points in $\mathbb{P}^{2}$ to lie on a curve of a certain degree. In higher projective space there is a result of Maroscia along similar lines [Ma, Theorem 2.3(2)]. This result requires that the points of $Z \subset \mathbb{P}^{n}$ satisfy a very weak general position assumption, and that the first difference of the Hilbert function take the value $n-h(h \geq 1)$ in each of $n-1$ consecutive degrees. The conclusion is that $Z$ has a large subset $Z_{1}$ lying on a rational normal curve in a linear subspace $\mathbb{P}^{n-h}$ of $\mathbb{P}^{n}$.

We will generalize this result in this section and in the next. For now we require only that $Z$ be a reduced set of points and that the first difference of the Hilbert function take the same value $s$ in each of $t w o$ consecutive degrees $d$ and $d+1$; our only constraints on the value of $s$ are that it be allowed in degree $d$ by Macaulay's growth condition, and that $d \geq s$ so that we have maximal growth. (Notice that we require only two degrees with this value.) Our result will be that $Z$ has a large subset $Z_{1}$ lying on a reduced curve $C$ of degree $s$, and that the first difference of the Hilbert function of $Z_{1}$ agrees with that of $C$ up to degree $d+1$ and with that of $Z$ afterwards. In the next section we will show that under various general position assumptions we can also show that $C$ is irreducible.

Remark 3.5. In the proof of the next result we will need the following facts from [GLP]. (We are grateful to E. Ballico for pointing out (2) to us.)

(1) (Theorem 1.1) If $X \subseteq \mathbb{P}^{n}$ is a reduced irreducible nondegenerate curve of degree $d$ then $\mathscr{J}_{X}$ is $(d+2-n)$-regular. 
(2) (Remark (1), p. 497) Let $X \subseteq \mathbb{P}^{n}$ be a reduced but not necessarily irreducible curve. Suppose $X$ has irreducible components $X_{i}$ of degree $d_{i}$, and that $X_{i}$ spans a $\mathbb{P}^{n_{i}} \subseteq \mathbb{P}^{n}$. Set

$$
m_{i}= \begin{cases}d_{i}+2-n_{i}, & \text { if } d_{i} \geq 2 \\ 1, & \text { if } d_{i}=1 \text { (i.e., if } X_{i} \text { is a line). }\end{cases}
$$

Then $X$ is $\left(\sum m_{i}\right)$-regular.

In the following theorem, "variety" does not necessarily imply "irreducible."

Theorem 3.6. Let $Y \subset \mathbb{P}^{r+1}$ be a reduced scheme of any dimension. Assume that for some $d, \Delta H(Y, d)=\Delta H(Y, d+1)=s$, where $d \geq s$. Then

(a) $\operatorname{dim} Y \leq 1$.

(b) $\left\langle\left(I_{Y}\right)_{\leq d}\right\rangle$ is the saturated ideal of a curve $V$ of degree $s$ (not necessurily unmixed). Furthermore, $V$ is reduced.

Let $C$ be the unmixed one-dimensional component of the reduced curve in (b). Let $Y_{1}$ be the subvariety of $Y$ on $C$ and $Y_{2}$ the "residual" subvariety.

(c) $\left\langle\left(I_{Y_{1}}\right)_{\leq d}\right\rangle=I_{C}$.

(d) $\operatorname{dim} Y_{2}=0$ and $H\left(Y_{1}, t\right)=H(Y, t)-\left|Y_{2}\right|$ for all $t \geq d-1$.

(e)

$$
\Delta H\left(Y_{1}, t\right)= \begin{cases}\Delta H(C, t), & \text { for } t \leq d+1 ; \\ \Delta H(Y, t), & \text { for } t \geq d .\end{cases}
$$

In particular, $\Delta H\left(Y_{1}, t\right)=s$ for all $s \leq t \leq d+1$.

Proof. (Observe that in particular we are claiming that the values of $\Delta H(C, t)$, and $\Delta H(Y, t)$ agree in degrees $d$ and $d+1$.) As usual let $J=\left(I_{Y}+(L)\right) /(L)$ for a general linear form $L$. Notice that the condition $d \geq s$ means that the $d$-binomial expansion of $s$ is $\left(\begin{array}{c}d \\ d\end{array}\right)+\cdots+\left(\begin{array}{c}d-s+1 \\ d-s+1\end{array}\right)$, and so we have maximal growth in degree $d$ (for the ring $S / J$ ). This also shows that $\Delta H(Y, t) \leq s$ for all $t \geq d$.

Now, $J_{d}$ is the degree $d$ part of the saturated ideal of a zeroscheme of degree $s$ in $\mathbb{P}^{r+1}$ (by maximal growth and Gotzmann's Persistence Theorem giving the Hilbert polynomial, and a "catch up" argument as in Lemma 1.4). Hence $\left(I_{Y}\right)_{\leq d}$ is the saturated ideal (Lemma 1.4) of a scheme $V$ in $\mathbb{P}^{r+1}$ having an unmixed one-dimensional component which is a curve $C$ of degree $s$. (C is not necessarily reduced or irreducible, a priori, so we cannot directly apply Remark 3.5 to $C$.) This proves (a) and the first half of (b).

Let $Y_{1}$ be the subvariety of $Y$ lying on $C$ and $Y_{2}$ the remaining subvariety of $Y$. (By the way we have defined $C, \operatorname{dim} Y_{2}=0$ trivially-this is the first part of (d).) Then $V$ is supported on $C, Y_{2}$ and possibly some other "ghost" points. As in the proof of Theorem 3.3, let $W$ be the subscheme of $V$ supported on $Y_{2}$ and let $X$ be the residual scheme. (This is well-defined by [ZS]: see the remark following Theorem 8 , Chapter IV, $\S 5$.) Notice that we again have $I_{W} \cap I_{X}=I_{V}$ and $W \cap X=\varnothing$. Then the same proof as in Theorem 3.3 gives $h^{1}\left(\mathscr{J}_{Y_{2}}(t)\right)=0$ for all $t \geq d-1$ and so $\Delta H\left(Y_{2}, t\right)=0$ for all $t \geq d$.

Consider the exact sequence

$$
0 \rightarrow I_{V} \rightarrow I_{C \cup Y_{2}} \rightarrow \frac{I_{C \cup Y_{2}}}{I_{V}} \rightarrow 0
$$

(respectively, the same sequence with $C \cup Y_{2}$ replaced by $C$ ). Again, the rightmost term is supported on a zeroscheme, so after sheafifying, twisting by 
$t \geq d-1$ and taking cohomology we get (by Proposition 1.6)

$$
\begin{aligned}
h^{1}\left(\mathscr{I}_{C \cup Y_{2}}(t)\right)=0 & \text { for all } t \geq d-1, \\
h^{1}\left(\mathscr{I}_{C}(t)\right)=0 & \text { for all } t \geq d-1 .
\end{aligned}
$$

We also have an exact sequence

$$
0 \rightarrow I_{Y} \cap I_{C} \rightarrow I_{Y} \oplus I_{C} \rightarrow I_{Y}+I_{C} \rightarrow 0 .
$$

If we sheafify this, twist by $t \geq d-1$ and take cohomology, we get (by $\left(1^{\prime}\right)$ ) the short exact sequence

$$
0 \rightarrow\left(I_{C \cup Y_{2}}\right)_{t} \rightarrow\left(I_{Y}\right)_{t} \oplus\left(I_{C}\right)_{t} \rightarrow\left(I_{Y_{1}}\right)_{t} \rightarrow 0 .
$$

Hence for $t \geq d-1$ we have

$$
H\left(C \cup Y_{2}, t\right)=H(Y, t)+H(C, t)-H\left(Y_{1}, t\right) .
$$

On the other hand, from the exact sequence

$$
0 \rightarrow \mathscr{I}_{C \cup Y_{2}} \rightarrow \mathscr{O}_{\mathbb{P}^{r+1}} \rightarrow \mathscr{O}_{C \cup Y_{2}} \rightarrow 0
$$

we get from $\left(1^{\prime}\right)$ that $H\left(C \cup Y_{2}, t\right)=h^{0}\left(\mathscr{O}_{C}(t)\right)+\left|Y_{2}\right|$ for $t \geq d-1$. From a similar sequence replacing $C \cup Y_{2}$ by $C$, we get from $\left(1^{\prime \prime}\right)$ that $h^{0}\left(\mathscr{O}_{C}(t)\right)=$ $H(C, t)$ for $t \geq d-1$.

Now, combining this information and substituting it in (2) we get

$$
H\left(Y_{1}, t\right)=H(Y, t)-\left|Y_{2}\right|
$$

for all $t \geq d-1$. This proves (d). In particular,

$$
\Delta H\left(Y_{1}, t\right)=\Delta H(Y, t) \text { for all } t \geq d .
$$

and this proves the second half of the Hilbert function claimed in (e).

Notice that we still have maximal growth for $\Delta H\left(Y_{1}, d\right)$ ! The ideal $\left(I_{Y_{1}}\right)_{\leq d}$ hence defines a scheme consisting of an unmixed curve $C_{1}$ of degree $s$ plus some zeroscheme. We first claim that this curve $C_{1}$ is just $C$.

Consider the ideals

$$
\frac{I_{Y_{1}}+(L)}{(L)} \supset \frac{I_{Y}+(L)}{(L)} \text {. }
$$

By (3), these have the same dimension in all degrees $\geq d$, hence are equal in those degrees. Therefore they define the same degree $s$ zeroscheme in the hyperplane defined by $L$. But they define, respectively, the hyperplane section of $C$ and of $C_{1}$; hence these hyperplane sections coincide. But $Y, Y_{1}$, and hence $C$ and $C_{1}$ did not depend on the choice of the general hyperplane, so $C$ and $C_{1}$ have infinitely many hyperplane sections which agree. Since they are unmixed, this proves $C=C_{1}$.

Now we claim that $\bar{I}=\left\langle\left(I_{Y_{1}}\right)_{\leq d}\right\rangle$ is precisely the ideal of $C$. (Hence this ideal is unmixed!) We know that $\bar{I}$ is a saturated ideal (Lemma 1.4). Certainly $\bar{I}_{i} \subseteq\left(I_{C}\right)_{i} \subseteq\left(I_{Z_{1}}\right)_{i}$ for all $i$. But the first and last agree in degree $\leq d$, so we have that $\bar{I}_{i}=\left(I_{C}\right)_{i}$ for all $i \leq d . \bar{I}$ is generated in degree $\leq \bar{d}$; if we prove that the same is true for $I_{C}$ then we will be done. Let $H$ be a general hyperplane and consider the exact sequence

$$
0 \rightarrow \mathscr{I}_{C}(d-2) \rightarrow \mathscr{I}_{C}(d-1) \rightarrow \mathscr{I}_{C \cap H}(d-1) \rightarrow 0 .
$$


Taking cohomology we get

$$
\cdots \rightarrow H^{1}\left(\mathscr{I}_{C \cap H}(d-1)\right) \rightarrow H^{2}\left(\mathscr{I}_{C}(d-2)\right) \rightarrow H^{2}\left(\mathscr{I}_{C}(d-1)\right) \rightarrow 0 .
$$

But $d \geq s$ and $C \cap H$ is a zeroscheme of degree $s$. Therefore

$$
h^{1}\left(\mathscr{I}_{C \cap H}(d-1)\right)=0
$$

which implies that $h^{2}\left(\mathscr{I}_{C}(d-2)\right)=0$. Therefore $I_{C}$ is $d$-regular, hence is generated in degree $\leq d$. Therefore $\left\langle\left(I_{Y_{1}}\right)_{\leq d}\right\rangle=I_{C}$ as desired. This proves (c) and the first half of $(e)$.

We now prove that $C$ is reduced. Observe that since $I_{Y_{1}} \supseteq I_{C_{\text {red }}} \supseteq I_{C}$, and since the ends agree in degree $\leq d+1$, we have that $\left(I_{C}\right)_{i}=\left(I_{C_{\text {red }}}\right)_{i}$ for all $i \leq d+1$. Also, $I_{C}$ is generated in degree $\leq d$. It is enough to prove that $I_{C_{\text {red }}}$ is generated in degree $\leq d$, and for this it is enough to show that $I_{C_{\text {red }}}$ is $d$-regular. This comes from Remark 3.5 plus the assumption that $d \geq s$.

This last statement in (e) will follow once we prove that $\Delta H(C, t)=s$ for $s \leq t \leq d+1$. This follows from the fact that $C$ is $s$-regular.

Finally, we prove that the scheme $V$ defined by the saturated $I_{V}=\left\langle\left(I_{Y}\right)_{\leq d}\right\rangle$ is reduced. We have seen that the one-dimensional component of $V$ is reduced. Notice that $H(V, d)=H(Y, d)$ and that $H(C, d)=H\left(Y_{1}, d\right)$. Hence by $(\mathrm{d})$ and $(\mathrm{e}), H(C, d)=H\left(Y_{1}, d\right)=H(V, d)-\left|Y_{2}\right|$. But $Y$ is reduced so $Y_{2}$ is a reduced set of points. We have $V \supseteq C \cup Y_{2}$, so $H(V, d) \geq H\left(C \cup Y_{2}, d\right)=$ $H(C, d)+\left|Y_{2}\right|$ (the last equality by $\left.\left(1^{\prime}\right)\right)$. Thus we have equality so $V=C \cup Y_{2}$ and we are done.

Somewhat surprisingly, an analog of Davis' Theorem [D, Theorem 4.1] even holds in this situation of higher codimension:

Corollary 3.7. Let $Y \subset \mathbb{P}^{r+1}$ be a reduced scheme of dimension $\leq 1$. Assume that for some $d \geq s, \Delta H(Y, d)=s$ and that the saturated ideal $\left\langle\left(I_{Y}\right)_{\leq d}\right\rangle^{\text {sat }}$ defines a curve $V$ of degree $s$. Then (a)-(e) of Theorem 3.6 continue to hold.

Proof. Let $\bar{I}=\left\langle\left(I_{Y}\right)_{\leq d}\right\rangle$ and let $\bar{J}=\left[\frac{\bar{I}+(L)}{(L)}\right]$, an ideal in $S=R /(L)$. Note that $H(S / \bar{J}, d)=s$ since $\bar{I}_{i}=\left(I_{Y}\right)_{i}$ for all $i \leq d$. However, the saturation of $\bar{J}$ is the ideal of the hyperplane section of $V$, hence it takes the value $s$ for all degrees $\geq d$. Therefore $\bar{J}$ agrees with the saturated ideal of $V \cap H$ in all degrees $\geq d$, and $H(S / \bar{J}, t)=s$ for all $t \geq d$.

This means that $H(S / \bar{J},-)$ has maximal growth in degree $d$, so the same proof as in Lemma 1.4 gives that $\left\langle\left(I_{Y}\right)_{\leq d}\right\rangle$ is saturated. Let $V$ be the subscheme of $\mathbb{P}^{r+1}$ defined by $\left\langle\left(I_{Y}\right)_{\leq d}\right\rangle$. The same proof as in Proposition 1.6 establishes that $\mathscr{I}_{V}$ is $d$-regular, and then the same proof as in Theorem 3.6 works in our context.

Conjecture 3.8. In the context of Theorem 3.6, if we assume only that $\Delta H(Y, d)$ has maximal growth then $V$ will still be a reduced scheme (of dimension computed by determining the Hilbert polynomial from the maximal growth).

Example 3.9. The assumption of maximal growth in Theorem 3.6 (or the weaker assumption in Corollary 3.7) was necessary in order to conclude that $C$ is reduced. That is, if $Z$ is a reduced set of points and if the degree $d$ component of $I_{Z}$ defines a curve $C, C$ may not be reduced. For example, consider a complete intersection of two cubics in $\mathbb{P}^{3}$ linking a double line $Y_{1}$ of genus -2 
to a smooth curve $Y_{2}$ of degree 7 and genus 3 . The two cubics in the complete intersection generate the degree 3 component of the ideal of $Y_{2}$. Hence if $Z$ consists of 2000 points on $Y_{2}$, the ideal of $Z$ in degree 3 defines a complete intersection scheme which is not reduced (since it has $Y_{1}$ as a component). Notice that $Z$ even has the Uniform Position Property (see $\S 4$ ).

This example also shows that it is possible to have a nonreduced curve $Y$ supported on some curve $C$, such that $I_{Y}$ is generated in degree $\leq m$ and $I_{Y}$ and $I_{C}$ agree in degree $\leq m$.

Corollary 3.10. Let $Z \subset \mathbb{P}^{r+1}$ be a reduced set of points with $\Delta H(Z, d)=$ $\left(\begin{array}{c}d+m-1 \\ d\end{array}\right)-\left(\begin{array}{c}d-k+m-1 \\ d-k\end{array}\right)$ and $\Delta H(Z, d+1)=\left(\begin{array}{c}d+m \\ d+1\end{array}\right)-\left(\begin{array}{c}d-k+m \\ d+1-k\end{array}\right)$, where $m \geq 3$ and $d \geq k$. Then $Z$ contains a subset $Z_{1}$ lying on a reduced hypersurface $Y$ of degree $k$ inside a $\mathbb{P}^{m}$ of $\mathbb{P}^{r+1}$. The first difference of the Hilbert function of $Z_{1}$ satisfies

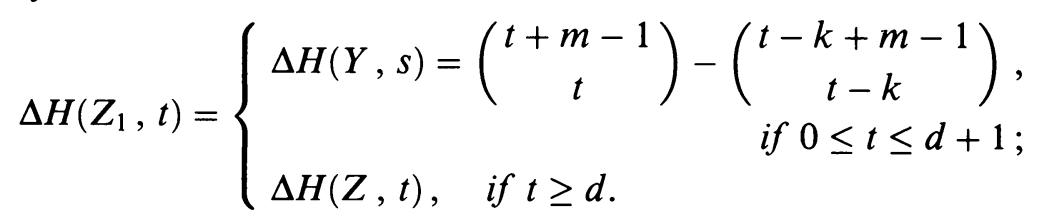

Proof. (Again we are claiming that the values of $\Delta H(Y, t)$ and $\Delta H(Z, t)$ agree in degrees $d$ and $d+1$.) As in Corollary 2.8, this is maximal growth. By Corollary 3.2, the degree $d$ (resp. $d+1$ ) component of $J=\left(I_{Z}+(L)\right) /(L)$ is in fact the degree $d$ (resp. $d+1$ ) component of a complete intersection of the desired form, of dimension $\geq m-1 \geq 1$. Thus if $V$ is, as usual, the scheme defined by the saturated ideal $\left\langle\left(I_{Z}\right)_{\leq d}\right\rangle$, the top-dimensional component $Y$ of $V$ has as its hyperplane section a complete intersection of dimension $\geq 1$ and hence $Y$ is itself a complete intersection of the claimed form. As before, $V$ then consists of $Y$ plus points. Let $Z_{1}$ be the subset of $Z$ on $Y$ and $Z_{2}$ the subset off $Y$.

Now the same proof used in Theorem 3.6 gives our result here. The only difference is that many of the more difficult steps in Theorem 3.6 actually come almost for free here, since we had to prove some things about the "mysterious" curve $C$ while we know that $Y$ is a complete intersection.

Remark 3.11. It is worth comparing Theorem 3.6 and Corollary 3.10. If we formally set $m=2$ in the hypothesis of Corollary 3.10 , we obtain the hypothesis of Theorem 3.6. However, if Corollary 3.10 applied in this case, the conclusion from Corollary 3.10 would be that the curve $C$ obtained in Theorem 3.6 was a plane curve of degree $s$. But that is obviously not the case in general (consider a large number of points on a rational normal curve). Thus, the assumption of maximal growth like that of Corollary 3.10 puts extremely stringent demands on the postulation of subsets.

Example 3.12. In Theorem 3.6, one cannot say more about the Hilbert function of $Y_{1}$ other than that it coincides with that of some reduced curve (so one can try to describe the possible Hilbert functions of reduced curves). One cannot even say precisely how many points are in $Y_{1}$. For instance, consider the Hilbert function $\Delta H: 132220 \ldots$. This can be realized by 8 points, 4 each on two skew lines, or it can be realized by 7 points on a plane conic and one point off that plane. 
Example 3.13. One cannot get a formula for the Hilbert function of the "residual" set of points as simple as that of Theorem 2.4 (or even Proposition 2.3). For instance, consider a reduced set of points $Z$ with Hilbert function having first difference 136330 . We have seen that a large subset $Z_{1}$ of $Z$ lies on a reduced curve of degree 3 . In the next section we will see that with a small general position assumption we can even deduce that this curve is a twisted cubic. However, if we try to subtract

$$
\begin{aligned}
& t: 01234 \\
& H(Z, t): 13633 \\
& H\left(Z_{1}, t\right): 13333
\end{aligned}
$$

we would obtain 3 in degree 2, which cannot be a first difference of any Hilbert function.

It does follow from Theorem 4.2 that there are exactly 3 points off the curve. However, one can check that these 3 points can be general or they can all lie on a line (provided that the line does not meet the twisted cubic).

\section{CONSEQUENCES OF MAXIMAL GROWTH WITH UNIFORMITY ASSUMPTIONS}

In this section we consider a reduced set of points $Z$ in $\mathbb{P}^{r+1}$ and observe some consequences of maximal growth of the first difference of the Hilbert function assuming some general position properties of the points.

We start in the situation of Theorem 3.6, where the first difference of the Hilbert function takes the same value in two consecutive degrees. Observe that if $Z \subset \mathbb{P}^{2}$ then this kind of growth corresponds to Davis' situation where a GCD results. Hence this is covered in $\S 2$, and we can assume that $Z$ is a least in $\mathbb{P}^{3}$.

Definition 4.1. A set of points in $\mathbb{P}^{n}$ is said to be in linear general position if no $n+1$ of them lie in a hyperplane in $\mathbb{P}^{n}$.

Theorem 4.2. Let $Z \subset \mathbb{P}^{r+1}$ be a reduced set of points, $r+1 \geq 3$. Assume that $\Delta H(Z, d)=\Delta H(Z, d+1)=s$ for some $d \geq s$. Then $\left\langle\left(I_{Z}\right)_{\leq d}\right\rangle=I_{C}$ where $C$ is a reduced curve of degree $s$ (by Theorem 3.6). Let $Z_{1}$ be the subset of $Z$ lying on $C$ and $Z_{2}$ the "residual" subset.

(a) If $s \leq r+1$ and no $s+1$ of the points lie in a $\mathbb{P}^{s-1}$ then $C$ is a rational normal curve in a $\mathbb{P}^{s}$ of $\mathbb{P}^{r+1}$. Moreover, there is an integer $p \geq d+2$ such that

$$
\Delta H\left(Z_{1}, t\right)= \begin{cases}1, & \text { if } t=0 \\ s, & \text { if } 1 \leq t \leq p-1 \\ \Delta H(Z, p), & \text { if } t=p \\ 0, & \text { if } t>p\end{cases}
$$

(where $0 \leq \Delta H(Z, p)<s$ ). In particular, $\left|Z_{1}\right|=s(p-1)+1+$ $\Delta H(Z, p)$.

(b) If the points of $Z$ are in linear general position and $r+1 \leq s<2(r+1)$ then $C$ is irreducible and

$$
\Delta H\left(Z_{1}, t\right)= \begin{cases}\Delta H(C, t), & \text { if } t \leq d+1 \\ \Delta H(Z, t), & \text { if } t \geq d+2\end{cases}
$$


In particular, $\left|Z_{1}\right| \geq r+2+d s+\sum_{i \geq d+2} \Delta H(Z, i)$.

Proof. Part (a) of this theorem is closely related to Maroscia's result [Ma, Theorem 2.3(2)]. As noted in $\S 2$, the difference is that [Ma] assumes $s \leq r$ and at least $r$ consecutive occurrences of the value $s$ in the first difference of the Hilbert function.

For either part of the theorem, we have from Theorem 3.6 that

$$
\Delta H\left(Z_{1}, t\right)= \begin{cases}\Delta H(C, t), & \text { if } t \leq d+1 \\ \Delta H(Z, t), & \text { if } t \geq d+2 .\end{cases}
$$

We begin with (a). Suppose that $C=C_{1} \cup \ldots \cup C_{\delta}$ are the irreducible components of $C$. We have $\sum_{i=1}^{\delta} \operatorname{deg} C_{i}=s \leq r$. Since no $s+1$ of the points lie in a $\mathbb{P}^{s-1}$ and since a reduced, irreducible curve of degree $j$ lies in a $\mathbb{P}^{j}$, we see that at most $\left(\operatorname{deg} C_{i}+1\right)$ points of $Z_{1}$ lie on $C_{i}$ for each $i$. Notice that $\left|Z_{1}\right|>2 s+1$ by information we have in the first difference of the Hilbert function. Then

$$
2 s+1<\left|Z_{1}\right| \leq \sum_{i=1}^{\delta}\left(\operatorname{deg} C_{i}+1\right)=\delta+\sum_{i=1}^{\delta} \operatorname{deg} C_{i}=\delta+s .
$$

Hence $s+1<\delta$, contradicting the fact that $\operatorname{deg} C=s$. Hence $C$ is irreducible of degree $s$. It cannot lie in a $\mathbb{P}^{s-1}$ since that would force more than $s+1$ points of $Z$ to lie in a $\mathbb{P}^{s-1}$. Therefore $C$ is a rational normal curve of degree $s$ spanning a $\mathbb{P}^{s}$.

Finally, the Hilbert function claimed in (a) follows since $\Delta H\left(Z_{1}, t\right) \leq s$ for all $t \geq d+2$ by maximal growth, and if it takes values $<s$ more than once then Castelnuovo's Lemma (cf. [Ma]) forces there to be a set of points which violate the condition that no $s+1$ of $Z$ can lie in a $\mathbb{P}^{s-1}$.

For (b), we again suppose that $C=C_{1} \cup \cdots C_{\delta}$ where $\sum_{i=1}^{\delta} \operatorname{deg} C_{i}=s<$ $2(r+1)$. For any $C_{i}$ of degree $<r+1$ we again have at most $\operatorname{deg} C_{i}+1$ of the points of $Z_{1}$ on $C_{i}$. If $C$ is not irreducible then at most one component of $C$ is nondegenerate. If no component is nondegenerate then exactly the same proof as in (a) works here. (The fact that $s \leq r$ was not needed.)

Now assume that $C$ has a component $C_{1}$ which is nondegenerate in $\mathbb{P}^{r+1}$, $r+1 \geq 3$. By Remark 3.5, $\operatorname{reg} C_{1} \leq\left(\operatorname{deg} C_{1}-1\right)$. If $C \neq C_{1}$ then there is a component $C_{\delta}$ which is degenerate. Let $C=Y \cup C_{\delta}$. It follows from Remark 3.5 and the fact that $C_{1}$ is nondegenerate and $r+1 \geq 3$ that

$$
\operatorname{reg} Y \leq \operatorname{deg} Y-1=s-\operatorname{deg} C_{\delta}-1 \leq d-\operatorname{deg} C_{\delta}-1 .
$$

We have seen that there are at most $\operatorname{deg} C_{\delta}+1$ points of $Z_{1}$ on $C_{\delta}$. By the bound on $\operatorname{reg} Y$, we can choose a form $F$ of degree $d-\operatorname{deg} C_{\delta}-1$ which contains $Y$ but not any of the points of $Z_{1}$ not on $Y$. Then by choosing $\operatorname{deg} C_{\delta}+1$ sufficiently general hyperplanes through these remaining points, we obtain a form of degree $d$ which vanishes on $Z_{1}$ but not on $C_{\delta}$, contradicting the fact (from the proof of Theorem 3.6) that $\left\langle\left(I_{Z_{1}}\right)_{\leq d}\right\rangle=I_{C}$.

Finally we prove the lower bound on the number of points of $Z_{1}$. If $H=\mathbb{P}^{r}$ is a general hyperplane, the points of $C \cap H$ are in linear general position, and hence the first difference of the Hilbert function of $\mathrm{C} \cap \mathrm{H}$ is

$$
1 \quad r s-r-10 \ldots
$$


Note that we are using the fact that $s-r-1<r$ so Castelnuovo's Lemma again applies. This means that the Hilbert function of $C \cap H$ is

$$
1 \quad r+1 \quad s \quad s \ldots
$$

and so $\Delta H(C, t) \geq s$ for $t \geq 2$ and $\Delta H(C, 1)=r+1$. Combining this with the formula for $\Delta H\left(Z_{1},-\right)$ in (b) gives the result.

Definition 4.3. A reduced, finite set of points $Z$ is said to have the Uniform Position Property (U.P.P.) if any two subsets of the same cardinality have the same Hilbert function; namely, if $Y \subset Z$ is a subset of cardinality $n$, then for all $t$

$$
H(Y, t)=\min \{H(Z, t), n\} .
$$

We have already seen, in Theorem 4.2, how the imposition of a small amount of uniformity on a set of points, with a given Hilbert function, can dramatically affect the disposition of a large subset of those points.

We now investigate how the much stronger hypothesis of uniform position influences these matters.

Lemma 4.4. Let $Z \subset \mathbb{P}^{r+1}$ be a reduced set of points with U.P.P. and suppose that the forms in $\left(I_{Z}\right)_{d}$ all have $F$, of degree $k$, as a common factor. Then

(a) $F$ is irreducible, and

(b) $\left(I_{Z}\right)_{t}=(F)_{t}$ for all $t \leq d$.

In particular, $F$ defines the hypersurface of least degree containing the points of $Z$.

Proof. By assumption, $\left(I_{Z}\right)_{d} \subseteq(F)_{d}$ and so $\left(I_{Z}\right)_{t} \subseteq(F)_{t}$ for all $t \leq d$. It follows that the forms of least degree in $I_{Z}$ have degree $\geq k$ and all have $F$ as a factor. Let $\alpha \geq k$ be this least degree.

Suppose $H(Z, \alpha)=b_{\alpha}$ and let $Y$ be a subset of $Z$ consisting of $b_{\alpha}$ points. By U.P.P. we must have

$$
H(Z, t)=H(Y, t)=H\left(\mathbb{P}^{r+1}, t\right) \text { for all } t<\alpha
$$

and $H(Y, t)=b_{\alpha}$ for all $t \geq \alpha$. Thus, by construction, $\left(I_{Z}\right)_{\alpha}=\left(I_{Y}\right)_{\alpha}$. Since $Y$ is a set of points in uniform position (in the sense of [GeMa]) we can apply Corollary 3.8 of that paper to assert that the generic element of $\left(I_{Y}\right)_{\alpha}$ is irreducible. Since all elements of $\left(I_{Y}\right)_{\alpha}$ have $F$ as a factor, we conclude that $F$ is irreducible and so $\alpha=k$.

Once we have $F$ in $I_{Z}$ it follows that $(F)_{t} \subseteq\left(I_{Z}\right)_{t}$ and hence we have equality for all $t \leq d$.

If we combine this lemma with Proposition 2.7 and Corollary 2.8 we immediately obtain

Corollary 4.5. Let $Z$ be a set of points in $\mathbb{P}^{r+1}$ with U.P.P. Let $L$ be a general linear form in $R=k\left[X_{0}, \ldots, X_{r+1}\right]$ and let $J=\left[I_{Z}+(L)\right] /(L)$ in $S=R / L$, so that $H(S / J, t)=\Delta H(Z, t)$.

Let $0<k$ be the potential $G C D$ of $J_{d}$ and suppose $H(S / J, d)$ has maximal growth. Then

(a) $H(S / J, d)=f_{r, k}(d)$ and $H(S / J, d+1)=f_{r, k}(d+1)$.

(b) $J_{d}=(\bar{F})_{d}$ and $J_{d+1}=(\bar{F})_{d+1}$ where $\bar{F}$ is a form of degree $k$ in $S$. 
(c) $\left(I_{d}\right)=(F)_{d}$ and $\left(I_{d+1}\right)=(F)_{d+1}$ where $F$ is an irreducible form of degree $k$ in $R$.

Example 4.6. A set of points $Z$ in $\mathbb{P}^{4}$ with Hilbert function

$$
\begin{array}{lllllll}
1 & 5 & 15 & 35 & 61 & 98 & 98 \ldots
\end{array}
$$

cannot have U.P.P. Indeed, consider the first difference of the Hilbert function,

$$
\begin{array}{lllllll}
1 & 4 & 10 & 20 & 26 & 37 & 0 \ldots
\end{array}
$$

The growth from 26 to 37 in these degrees is maximal, and the potential GCD is 2 . Then Corollary 4.5 says that $Z$ must lie on a quadric hypersurface, and clearly that is to the case with this Hilbert function.

Theorem 4.7. Let $Z \subset \mathbb{P}^{r+1}$ be a reduced finite set of points with U.P.P. Assume that $\Delta H(Z, d)=\Delta H(Z, d+1)=s, d \geq s$. Then there exists a reduced, irreducible curve $C$ of degree $s$ such that

(a) $Z \subset C$;

(b) $I_{C}=\left\langle\left(I_{Z}\right)_{\leq d}\right\rangle$;

(c) $\Delta H(Z, t)=\Delta H(C, t)$ for all $t \leq d+1$.

Proof. If $r+1=2$, this is covered in $\S 2$, so we assume $r+1 \geq 3$. By Theorem 3.6, we know that there exists a reduced curve $C$ containing a subset $Z_{1} \subseteq Z$, such that $I_{Z_{1}}$ agrees with $I_{C}$ up to degree $d+1$. Thus by U.P.P., $I_{Z}$ agrees with $I_{C}$ and hence with $I_{Z_{1}}$ up to degree $d+1$. But theorem 3.6 says that $\Delta H\left(Z_{1}, t\right)=\Delta H(Z, t)$ for $t \geq d$, so we get that $Z=Z_{1}$. This proves (a), (b), and $(\mathrm{c})$.

It remains only to prove that $C$ is irreducible. If $C$ contains a component which is degenerate, the same proof as in Theorem 4.2 works here; hence we may assume that all the components of $C$ are nondegenerate, and so of degree $\geq r+1$. Let $C_{1}, \ldots, C_{\delta}$ be the components of $C$ and write $s_{i}=\operatorname{deg} C_{i}$. By Remark 3.5, each component $C_{i}$ has regularity $\leq s_{i}+2-(r+1)$.

Let $Z_{i}$ be the subset of $Z$ lying on $C_{i}$. Since $Z_{i} \subset C_{i} \subset C$, we get that $H\left(Z_{i}, t\right) \leq H\left(C_{i}, t\right) \leq H(C, t)$ for all $t$. Notice that $H(C, t)=H(Z, t)$ for all $t \leq d+1$. Thus for any $t \leq d+1$ for which $H\left(C_{i}, t\right)<H(C, t)$, we have $H\left(Z_{i}, t\right)<H(Z, t)$. For such a $t$, then, U.P.P. implies that $\left|Z_{i}\right|=H\left(Z_{i}, t\right)<$ $H(Z, t)$.

Notice that in any case the inequality $H\left(C_{i}, t\right)<H(C, t)$ is satisfied for $t=\operatorname{reg} C_{i}$ since in that degree the homogeneous component of $I_{C_{i}}$ cuts out $C_{i}$. Thus

$$
\left|Z_{i}\right|=H\left(Z_{i}, s_{i}+1-r\right)<H\left(Z, s_{i}+1-r\right),
$$

and so

$$
|Z| \leq \sum\left|Z_{i}\right|<H\left(Z, s_{1}+1-r\right)+\cdots+H\left(Z, s_{\delta}+1-r\right) .
$$

By (1), the points of $Z_{i}$ impose independent conditions on forms of degree $s_{i}+1-r$. Now, choosing any point $P \in Z$, omit it from any $Z_{i}$ in which it may occur. Then choose a form $F_{i}$ of degree $s_{i}+1-r$ vanishing on all points of $Z_{i}$ other than $P$. The product $F=F_{1} \cdots F_{\delta}$ vanishes at all the points of $Z$ but $P$, so $Z$ imposes independent conditions on forms of degree $\left(s_{1}+1-r\right)+\cdots+\left(s_{\delta}+1-r\right)=s+\delta(1-r)<s \leq d$. But $H(Z, d) \leq|Z|-s$ so this is impossible. 
Remark 4.8. Thanks to Corollary 3.7, we could have obtained the results of Theorem 4.7 assuming only that the value of $\Delta H(Z,-)$ is $s$ in degree $d$ and that the homogeneous component of the ideal in degree $d$ defines a curve of degree $s$.

Conjecture 4.9. Let $Z$ be a finite set of points in $\mathbb{P}^{r+1}$ with U.P.P. and assume that $\Delta H(Z,-)$ has maximal growth in degree $d$. Then the scheme $V$ defined by the homogeneous component of the ideal of $Z$ in degree $d$ is not only reduced but irreducible as well, and $Z \subset V$. (See also Conjecture 3.8.)

Example 4.10. Let $Z$ be a finite set of points in $\mathbb{P}^{3}$ with Hilbert function

$$
\begin{array}{lllllll}
1 & 4 & 10 & 15 & 19 & 23 & 23 \ldots
\end{array}
$$

Then $Z$ cannot have U.P.P. Indeed, consider the first difference of the Hilbert function,

$$
\begin{array}{lllllll}
1 & 3 & 6 & 5 & 4 & 4 & 0 \ldots
\end{array}
$$

If $Z$ had U.P.P., then, by Theorem 4.7, $Z$ must lie on a reduced irreducible curve $C$ of degree 4, and its Hilbert function must agree with that of $C$ up to degree 5 . In particular, $C$ cannot lie on a surface of degree 2 , whereas any reduced, irreducible curve of degree 4 in $\mathbb{P}^{3}$ does lie on a quadric.

Remark 4.11. As is well-known, restrictions on the Hilbert function of points with U.P.P. in $\mathbb{P}^{r}$ give restrictions on the postulation of a general hyperplane section of a curve in $\mathbb{P}^{r+1}$. (For a good example of how Davis' theorem about points in $\mathbb{P}^{2}[\mathrm{D}$, Theorem 4.1] can be used to obtain information about curves, see [ES]).

In a similar way, the results of the present paper (especially $\S 4$ ) should have implications for curves. We have begun to investigate this idea, and the results of that study will be the subject of another paper.

\section{REFERENCES}

[D] E. D. Davis, Complete intersections of codimension 2 in $\mathbb{P}^{r}$ : The Bezout-Jacobi-Segre theorem revisited, Rend. Sem. Mat. Univ. Politec. Torino 432 (1985), 333-353.

[EH] D. Eisenbud and J. Harris, Curves in projective space, Les Presses de L'Université de Montréal, 1982.

[ES] P. Ellia and S. Strano, Sections planes et majoration du genre des courbes gauches, Complex Projective Geometry, Lecture Notes Series, London Math. Soc., no. 179, 1992.

[GeMa] A. V. Geramita and P. Maroscia, The ideal of forms vanishing at a finite set of points in $\mathbb{P}^{n}$, J. Algebra 90 (1984), 528-555.

[GMR] A. V. Geramita, P. Maroscia, and L. Roberts, The Hilbert function of a reduced $K$-algebra, J. London Math. Soc. (2) 28 (1983), 443-452.

[Go] G. Gotzmann, Eine Bedingung für die Flachheit und das Hilbertpolynom eines graduierten Ringes, Math. Z. 158 (1978), 61-70.

[Gr] M. Green, Restrictions of linear series to hyperplanes, and some results of Macaulay and Gotzmann, Lecture Notes in Math., vol. 1389, Algebraic Curves and Projective Geometry (Proceedings, Trento, 1988), Springer-Verlag, 1989.

[GLP] L. Gruson, R. Lazarsfeld, and C. Peskine, On a theorem of Castelnuovo and the equations defining space curves, Invent. Math. 72 (1983), 491-506.

[M] F. S. Macaulay, Some properties of enumeration in the theory of modular systems, Proc. London Math. Soc. 26 (1927), 531-555. 
[Ma] P. Maroscia, Some problems and results on finite sets of points in $\mathbb{P}^{n}$, Lecture Notes in Math., vol. 997, Algebraic Geometry-Open Problems (Proceedings, Ravello 1982), Springer, 1983.

[Mu] D. Mumford, Lectures on curves on an algebraic surface, Ann. of Math. Stud., no. 59, Princeton Univ. Press, Princeton, N.J., 1966.

[Ra] G. Raciti, Sulla funzione di Hilbert di un sottoschema zero-dimensionale di $\mathbb{P}^{3}$, Ann. Univ. Ferrara Sez. VII Sci. Mat. 35 (1989), 99-112.

[Ro] L. Robbiano, An introduction to the theory of Hilbert functions, The Curves Seminar at Queen's, vol. 7, Queen's Papers in Pure and Appl. Math., No. 85, 1990, pp. B1-B26.

[S] R. Stanley, Hilbert functions of graded algebras, Adv. in Math. 28 (1978), 71-82.

[ZS] O. Zariski and P. Samuel, Commutative algebra, vol. I, Van Nostrand, Princeton, N.J., 1967.

Dipartimento di Matematica, Università di Genova, 16132 Genova, Italy

E-mail address: bigatti@dima.unige.it

Department of Mathematics and Statistics, Queen's University, Kingston K7L 3N6, CANADA

E-mail address: geramita@qucdn.queensu.ca

Department of Mathematics, University of Notre Dame, Notre Dame, Indiana 46556

E-mail address: juan.c.migliore.1@nd.edu 